\title{
Disruption of CCR1-mediated myeloid cell accumulation suppresses colorectal cancer progression in mice
}

\section{$\operatorname{AUTHOR}(\mathrm{S})$ :}

Kiyasu, Yoshiyuki; Kawada, Kenji; Hirai, Hideyo; Ogawa, Ryotaro; Hanada, Keita; Masui, Hideyuki; Nishikawa, Gen; ... Kai, Masayuki; Taketo, Mark Makoto; Sakai, Yoshiharu

\section{CITATION:}

Kiyasu, Yoshiyuki ... [et al]. Disruption of CCR1-mediated myeloid cell accumulation suppresses colorectal cancer progression in mice. Cancer letters 2020, 487: 53-62

\section{ISSUE DATE:}

2020-09-01

URL:

http://hdl.handle.net/2433/253735

\section{RIGHT:}

(C) 2020. This manuscript version is made available under the CC-BY-NC-ND 4.0 license

http://creativecommons.org/licenses/by-nc-nd/4.0/.; The full-text file will be made open to the public on 1 September 2021 in accordance with publisher's 'Terms and Conditions for Self-Archiving'.; この論文は出版社版でありません。引 用の際には出版社版をご確認ご利用ください。; This is not the published version. Please cite only the published version. 


\section{Disruption of CCR1-mediated myeloid cell accumulation suppresses colorectal cancer progression in mice}

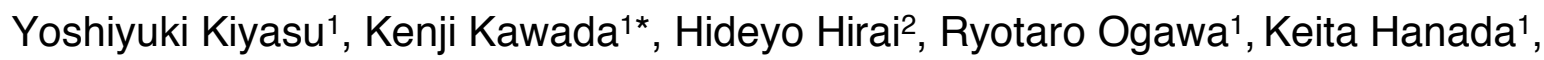
Hideyuki Masui ${ }^{1}$, Gen Nishikawa ${ }^{1}$, Takamasa Yamamoto ${ }^{1}$, Rei Mizuno ${ }^{1}$, Yoshiro Itatani', Masayuki Kai ${ }^{3}$, Makoto Mark. Taketo ${ }^{4}$, Yoshiharu Sakai ${ }^{1}$

Authors' Affiliations: Departments of Surgery ${ }^{1}$ and Clinical Laboratory Medicine ${ }^{2}$, Graduate School of Medicine, Kyoto University, Kyoto 606-8507, Japan.

${ }^{3}$ Oncology Research Laboratories, Oncology R\&D Unit, R\&D Division, Kyowa Kirin Co., Ltd.3-6-6 Asahi-machi, Machida, Tokyo, 194-8533, Japan

${ }^{4}$ Division of Experimental Therapeutics, Graduate School of Medicine, Kyoto University, Yoshida-Konoe-cho, Sakyo-ku, Kyoto 606-8501, Japan

Corresponding author: Kenji Kawada, Department of Surgery, Graduate School of Medicine, Kyoto University, 54 Shogoin- Kawara-cho, Sakyo-ku, Kyoto, Japan, 606-8507. Phone: +81-75-366-7595; FAX: +81-75-366-7642; E-mail: kkawada@kuhp.kyoto-u.ac.jp

Running title: Disruption of CCR1 suppresses colorectal cancer progression

Potential Conflict of Interest: MK is an employee of Kyowa Kirin Co., Ltd. All other authors declare that they have no potential conflicts of interest.

Word Counts: 2862 words

Figures and tables: 5 figures

Supplementary figures and tables: Supplementary 3 figures and 2 tables 


\begin{abstract}
Tumor-stromal interaction is implicated in tumor progression. Although CCR1 expression in myeloid cells could be associated with pro-tumor activity, it remains elusive whether disruption of CCR1-mediated myeloid cell accumulation can suppress tumor progression. Here, we investigated the role of CCR1 depletion in myeloid cells in two syngeneic colorectal cancer mouse models: MC38, a transplanted tumor model and CMT93, a liver metastasis model. Both cells induced tumor accumulation of CCR1+ myeloid cells that express MMP2, MMP9, iNOS, and VEGF. Lack of the Ccr1 gene in host mice dramatically reduced MC38 tumor growth as well as CMT93 liver metastasis. To delineate the contribution of CCR1 + myeloid cells, we performed bone marrow (BM) transfer experiments in which sub-lethally irradiated wild-type mice were reconstituted with BM from either wild-type or $\mathrm{Ccr1}^{-/-}$mice. Mice reconstituted with $\mathrm{Ccr}^{-1-} \mathrm{BM}$ exhibited marked suppression of MC38 tumor growth and CMT93 liver metastasis, compared with control mice. Consistent with these results, administration of a neutralizing anti-CCR1 monoclonal antibody, KM5908, significantly suppressed MC38 tumor growth and CMT93 liver metastases. Our findings highlight the importance of the application of CCR1 blockade as a therapeutic strategy.
\end{abstract}

Key words: colorectal cancer, CCR1, tumor microenvironment, myeloid cell,

Abbreviations: CRC, colorectal cancer; TME, tumor microenvironment; TAN, tumor-associated neutrophil; TAM, tumor-associated macrophage; MDSC, myeloid-derived suppressor cell; HCC, hepatocellular carcinoma; mAb, monoclonal antibody; CMT93-luc, CMT93 with luciferase; BM, bone marrow; IHC, immunohistochemistry; MMP, matrix metalloproteinase; iNOS, inducible nitric oxide synthase; VEGF, vascular endothelial growth factor; Treg, regulatory T cell; PDCA, pancreatic ductal cell carcinoma. 


\section{Introduction}

Colorectal cancer (CRC) is one of the leading causes of cancer-related deaths globally. Further understanding of the $\mathrm{CRC}$ biology can help establish new therapeutic modalities. Recently, the tumor-stromal interaction within the tumor microenvironment (TME) has emerged as a potential therapeutic target. For example, infiltration of CD3 ${ }^{+}$ and $C D 8^{+} T$ cells into the $C R C$ tissues results in a favorable prognosis of Stage I-III patients [1]. Immune checkpoint blockade therapy has provided clinical benefit to microsatellite instability-high CRC patients [2]. Recent data have shown that several types of myeloid cells, including tumor-associated neutrophils (TANs), tumor-associated macrophages (TAMs), and myeloid-derived suppressor cells (MDSCs), play critical roles in tumor progression by releasing a number of cytokines and chemokines [3, 4]. Unlike cancer cells, host cells are genetically stable and represent promising therapeutic targets, since they are less likely to develop drug resistance [5].

Chemokines are a family of chemoattractant polypeptides cytokines that regulate the migration and positioning of immune cells through interaction with cognate $G$ protein-coupled receptors, and are involved in multiple TME-related processes [6, 7]. We and others have shown that loss of SMAD4 from CRC cells promotes expression of mouse CCL9 or human CCL15 (the human orthologue of mouse CCL9), which causes the recruitment of $\mathrm{C}-\mathrm{C}$ motif chemokine receptor 1 (CCR1)-expressing myeloid cells to facilitate tumor invasion and metastasis [8-13]. In human CRC samples, most CCR1+ myeloid cells recruited to CRCs were neutrophils and MDSCs [11-13]. Recently, other groups have also reported that recruitment of $\mathrm{CCR} 1^{+}$myeloid cells can promote distant metastasis to the liver and lungs in hepatocellular carcinoma (HCC), breast cancer, and thymoma [14-16].

Local microenvironmental conditions can affect the polarization of myeloid cells toward a pro- or anti-tumor state [17-19]. The clinical significance of myeloid cell infiltration into CRC tissues remains to be elucidated. Therefore, in the present study, we investigated the possibility of CCR 1 signaling blockade as a therapeutic strategy for CRC using two syngeneic mouse models (MC38 and CMT93). Here we present the results of the antitumor effects of CCR 1 depletion by the genetic deficiency of CCR1 or by pharmacologically blocking CCR 1 with a neutralizing anti-CCR 1 monoclonal antibody 
(mAb), which provides a rationale for preventing the accumulation of $\mathrm{CCR} 1^{+}$myeloid cells in cancer therapy. 


\section{Materials and Methods}

\subsection{Mice and tumor cell lines}

In in vivo experiments, C57BL/6 wild-type mice or C57BL/6 Ccr ${ }^{1 /-}$ mice [8-10] were used at 8 to 10-week-old. Mouse colon cancer cell lines with C57BL/6 background, MC38 and CMT93 with luciferase (CMT93-luc) $[9,10]$, were cultured at $37^{\circ} \mathrm{C}$ in DMEM with $10 \%$ fetal calf serum under $5 \% \mathrm{CO}_{2}$. The sample size of in vivo experiments was determined on the basis of prior experience with similar studies [9-13]. A statistical analysis was performed with a two-tailed, two-sample $t$ test or Mann-Whitney $U$ test with an $\alpha$ of 0.05 and a power of 0.8 to detect differences between groups. All groups were randomized, and primary endpoints were tumor size of MC38 transplanted tumors or IVIS score of CMT93 liver metastases. All procedures were performed in accordance with institutional protocols approved by the Animal Care and Use Committee of Kyoto University.

\subsection{Bone marrow (BM) transplantation}

Donor's BM cells harvested from either C $\mathrm{Cr}^{-1-}$ mice or its littermate wild-type C57BL/6 mice were injected into the tail vein of recipient wild-type C57BL/6 mice that had been irradiated with 8-Gy gamma-rays half a day before. 12 weeks after BM transplantation, the recipient mice were inoculated with tumor cells.

\subsection{Transplanted tumor model}

$1.0 \times 10^{6}$ cells of MC38 suspended with $50 \mu \mathrm{l}$ sterile PBS and $50 \mu \mathrm{l}$ Matrigel (Corning) were subcutaneously injected into the dorsum of mice. The size of the transplanted subcutaneous tumors was measured as volume using the formula $\left(L_{1} \times L_{1} \times L_{2}\right) / 2$, where $L_{1}$ is the shortest diameter and $L_{2}$ is the longest diameter. On days $46-49$ post-injection, transplanted subcutaneous tumors were harvested for histological analyses.

\subsection{Experimental liver metastasis model}

$1.5 \times 10^{6}$ cells of CMT93-luc suspended in $100 \mu$ sterile PBS were injected into the hilum of the spleen. The spleen was removed $1 \mathrm{~min}$ after tumor cell injection to prevent splenic tumor formation. For in vivo bioluminescence imaging, $3 \mathrm{mg}$ of VivoGlo ${ }^{\mathrm{TM}}$ Luciferin (Promega) was injected intraperitoneally into anesthetized tumor-bearing mice 
10 min before imaging. Bioluminescence from the CMT93-luc cells was monitored on days 1, 4, 7, 14, 21 post-injection, using a Xenogen IVIS system (Xenogen Corporation). On day 21 post-injection, the liver was harvested for histological analyses.

\subsection{RT-PCR, quantitative RT-PCR and PCR}

Total RNA was extracted using the High Pure RNA Isolation Kit (Roche Diagnostics). Complementary DNAs was generated using ReverTra ace qPCR RT kit (Toyobo Co. Ltd.,). The following sets of primers were used for RT-PCR: Ccl3 forward, 5'-GGTCTCCACACTGCCCTT-3'; Ccl3 reverse, 5'-TCAGGCATTCAGTTCCAGGTC-3'; Ccl4 forward, 5'-GAAGCTCTGCGTGTCTGCCCT-3'; Ccl4 reverse, 5'-ACTCCAAGTCACTCATGTACT-3'; Ccl5 forward, 5'-ATGAAGATCTCTGCAGCTGCC-3'; Ccl5 reverse, 5'-CTAGCTCATCTCCAAATAGTT-3'; Ccl6 forward, 5'-AGGATGAGAAACTCCAAGACTG-3'; Ccl6 reverse, 5'-TCAAGCAATGACCTTGTTCCCA-3'; Ccl7 forward, 5'-GCTCATAGCCGCTGCTTTC-3'; Ccl7 reverse, 5'-GCTTTGGAGTTGGGGTTTTC-3'; Ccl9 forward, 5'-ATGAAGCCTTTTCATACTGCCCTC-3'; Ccl9 reverse, 5'-TTATTGTTTGTAGGTCCGTGGTTG-3'; and Gapdh forward, 5'-CCTTCATTGACCTCAACTAC-3'; Gapdh reverse, 5'-TGGGCCCTCAGATGCCTGCT-3'. For quantitative RT-PCR, the following sets of primers were used: Ccr1 forward, 5'-TGGACAAAATACTCTGGAAACACA-3'; Ccr1 reverse, 5'-TGTGAAATCTGAAATCTCCATCC-3'; and Gapdh forward, 5'-TGTCCGTCGTGGATCTGAC-3'; Gapdh reverse, 5'-CCTGCTTCACCACCTTCTTG-3'. For genotypic DNA analysis of Ccr1, the following sets of primers were used: Ccr1 forward, 5'-TCCTCAGCAAAGGATGGAGA-3'; Ccr1 reverse, 5'-CAGACGCACGGCTTTGACCTTCTT-3'.

\subsection{Immunohistochemistry (IHC) and immunofluorescence analysis}

For IHC, formalin-fixed, paraffin-embedded sections were stained with the respective primary antibodies (Supplementary Table 1) by the avidin-biotin immunoperoxidase method. We quantified the densities of $\mathrm{CCR}^{+}, \mathrm{Ly}_{6 \mathrm{G}}{ }^{+}, \mathrm{CD}^{+}$, and $\mathrm{FOXP3}{ }^{+}$cells at the peritumoral region. As the tumoral microvessel density, we quantified the densities of 
CD31+ endothelial cells within the tumors. The average of $10-20$ fields $\left(0.1 \mathrm{~mm}^{2}\right)$ analyzed per one sample, and three researchers ( $\mathrm{YK}, \mathrm{KH}$, and $\mathrm{HM}$ ) evaluated IHC samples without prior knowledge of other data. The slides with different evaluations among them were reinterpreted followed by a conclusive judgment. For immunofluorescence analysis, tissue sections following antigen retrieval were incubated with primary antibodies (Supplementary Table 1 ) overnight at $4^{\circ} \mathrm{C}$, followed by the 2nd antibodies. Representative photos were taken using a fluorescence microscope (BZX-710; Keyence).

\subsection{Flow cytometric analysis}

Flow cytometric analysis and cell sorting were performed using BD FACSCanto II and FACSAria III (BD Biosciences), as previously described [10]. Cells were stained with anti-CD11b Ab (clone M1/70), anti-Ly6G Ab (clone 1A8), and anti-CD115 Ab (clone AFS98) (Supplementary Table 2). Anti-CCR1 Ab, KM5908, was provided from Kyowa Kirin Co., Ltd. Propidium iodide (PI) was used to eliminate dead cells. Data were analyzed with the FlowJo software (BD Biosciences).

\subsection{Production of novel anti-CCR1 mAb, KM5908}

cDNAs encoding human and mouse CCR1 were synthesized (GenScript) and cloned into Tol2 transposon vector Tn-pMug-Hygro [20]. The expression vector was co-transfected with Tol2 transposase expression vector TPEX-pMug [20] into CHO-S cells (ThermoFisher Scientific) and a rabbit cell line RM-33 (JCRB) [21]. Stable cell lines over expressing human CCR1 were established through hygromycin selection. Ccr $1^{1-1}$ mice (B6.129S4-Ccr1 ${ }^{\mathrm{tm} 1 \mathrm{Gao}} \mathrm{N} 10+\mathrm{N} 5$, purchased from Taconic) [22] were immunized with the human CCR1-expressing cell lines three times. After checking serum antibody titers by flow cytometry, spleen cells were fused with P3-U1 myeloma cells (ATCC) by the PEG method and selected in the HAT-medium. The hybridomas were first screened based on binding activity to human and mouse CCR1, then selected by inhibitory activity of chemotaxis of human monocytic cell line THP-1 (ATCC) toward CCL15 gradient. As a result, nine hybridomas producing anti-CCR 1 inhibitory $A b$ were established and $\mathrm{mAbs}$ were purified from the supernatants by affinity purification using Protein $G$ Sepharose 4 
Fast Flow (GE Healthcare). One of the mAbs established, named KM5908, was selected for in vivo study.

\subsection{THP-1 chemotaxis assay}

Chemotaxis assay was performed as follows. THP-1 cells were cultured in RPMI medium (Nakalai Tesque) supplemented with 10\% FBS and $5 \mathrm{mM}$ all-trans retinoic acid (Wako) for three days to enhance CCR1 expression. Then the cells were harvested and resuspended in RPMI with $1 \%$ FBS at $1 \times 10^{6}$ cells $/ \mathrm{mL}$. A hundred microliters of the cell suspension was incubated for 1 hour with the hybridoma supernatant or purified antibody then placed in the upper chamber of Transwell with $5.0 \mu \mathrm{m}$ pore (Corning). In lower chamber $1 \mathrm{ng} / \mathrm{mL}$ of recombinant human CCL15 (R\&D Systems) was added as chemoattractant. The cells were cultured for 4 to 6 hours for migration. Cells migrated to lower chamber were quantified by using Celltiter-Glo assay (Promega).

\subsection{Binding analysis}

Specific binding of KM5908 to human and mouse CCR1 was confirmed by flowcytometric analysis. CCR1-expressing CHO-S cells were stained with KM5908 or isotype Control (R\&D Systems) and then with secondary antibody anti-mouse IgG Alexa Fluor 647 (ThermoFisher Scientific). Samples were analyzed by BD FACSCanto II flow cytometer and FlowJo software (BD Biosciences).

\subsection{1, Drug treatment}

The isotype control for KM5908 was purchased from Biolegend. Anti-PD1 mAb (clone RMP 1-14) and its isotype control were purchased from BioXCell. These antibodies were diluted in PBS at $1 \mathrm{mg} / \mathrm{ml}$. Anti-CCR1 mAb and its isotype control were injected subcutaneously at a dose of $10 \mu \mathrm{g} / \mathrm{g}$ per injection. The PD-1 and its isotype control were injected intraperitoneally at the same dose. Anti-CCR1 treatment was started from 3 days before tumor inoculation and then continued twice a week to the end of the analysis. As for anti-PD1 mAb treatment, mice anti-PD-1 or its isotype control was given intraperitoneally on days 7, 11, 14, 18. Eight-week-old C57BL/6 mice were obtained from Japan SLC (Hamamatsu, Japan). 


\subsection{Statistical analysis}

All results were confirmed using at least three independent experiments, and data from one representative experiment were presented. Statistical significance was evaluated with the Student's $t$-test in the subcutaneous transplanted tumor model and the Mann-Whitney $U$ test in the liver metastasis model. Categorical data were examined using the Fisher's exact test. All statistical analyses were two-tailed and performed with JMP Pro 14 (SAS Institute Inc). $P$ value $<0.05$ was considered as statistically significant. Differences between groups were considered significant if $P$-values were $<0.05$. Analyzed values were expressed as mean values \pm standard error of the mean (SEM). 


\section{Results}

\subsection{Accumulation of $C C R 1^{+}$myeloid cells around the peritumoral stroma of CRC}

Although we and others have demonstrated that the recruitment of CCR1+ myeloid cells to SMAD4-deficient CRCs promotes tumor invasion and metastasis [8-13], the role of $\mathrm{CCR} 1^{+}$myeloid cells in the TME remains undefined. We first investigated CCR1 expression in murine immune cells in the peripheral blood and bone marrow (BM) by flow cytometric analysis and quantitative RT-PCR, and found that CCR1 was highly expressed on neutrophils (CD11b+/ Ly6G+) and monocytes (CD11b+/CD115+), while it was barely expressed on lymphocytes (CD11b/FSClow/SSClow) (Fig. 1A, B). In this study, we investigated the role of CCR1 deletion in the TME of CRC using two syngeneic C57BL/6 mouse models: MC38 cells (a transplanted tumor model) and CMT93 cells (a liver metastasis model). Among several CCR1 ligands, Cc/9 mRNA was abundantly produced in both MC38 and CMT93 cells, while low levels of Cc/7 mRNA was found in MC38 cells (Fig. 1C). Immunohistochemical (IHC) analysis also confirmed that both lines of $\mathrm{CRC}$ cells expressed the Ccl9 protein in vivo, whereas the surrounding stromal cells did not (Fig. 1D, E). As a transplanted tumor model, we injected MC38 cells subcutaneously into the wild-type and $\mathrm{Ccr}^{-/-}$mice. There were no significant differences in the distribution of circulating blood cells between the wild-type and $\mathrm{CCr}^{-1-}$ mice (Supplementary Fig. S1A). IHC analysis indicated that CCR1+ myeloid cells accumulated around the MC38 tumors in wild-type, but not in $\mathrm{Ccr1}^{-/-}$mice (Fig. 1D). We further characterized CCR1 + myeloid cells by double immunofluorescence staining, and found that the majority of these CCR1 + cells expressed matrix metalloproteinase (MMP)2, MMP9, inducible nitric oxide synthase (iNOS), and vascular endothelial growth factor (VEGF) (Fig. 1F). The growth of MC38 transplanted tumors was significantly reduced in the $\mathrm{Crr1}^{-/-}$mice compared with that in the wild-type mice (Fig. 2A, B). On day 49 post-injection, the tumor size in the wild-type mice was $657 \pm 98 \mathrm{~mm}^{3}$, whereas that in the $\mathrm{Ccr1}^{-1-}$ mice was $353 \pm 78 \mathrm{~mm}^{3}(P<0.05)$. On the other hand, intrasplenic injection of CMT93 cells can cause efficient metastasis of tumor cells into the liver [9, 10]. As a model of liver metastasis, we injected luciferase (luc)-expressing CMT93 cells into the spleen of the wild-type and $\mathrm{Ccr1}^{-/-}$mice, and then monitored tumor metastasis to the liver using bioluminescence, which enabled quantification of tumor cells within the liver by photon counting. As anticipated, histological analysis indicated that CCR1+ myeloid cells 
accumulated around the metastatic lesions in the liver of the wild-type mice (Fig. 1E), and that the majority of CCR1 ${ }^{+}$cells expressed MMP2, MMP9, iNOS, and VEGF (Fig. $1 \mathrm{G})$. Regarding the quantification of the metastasized tumor cells, liver luciferase intensities in the $\mathrm{Ccr}^{-/-}$mice were significantly weaker compared with those in the wild-type mice (Fig. 2C, D). On days 14 and 21 post-injection, there was a significant reduction of the liver luminescence in the $\mathrm{Ccr}^{-1-}$ mice compared with that in the wild-type mice $(P<0.05)$, which took place only after day 7 post-injection. We dissected the liver on day 21 post-injection, and examined macroscopic metastatic foci. Although wild-type mice formed macroscopic foci in 83\% (10 of 12), Ccr1 $^{-/-}$mice formed only in 33\% (3 of 9) $(P<0.05)$ (Supplementary Fig. S1B).

\subsection{Lack of CCR1 in hematopoietic myeloid cells leads to suppression of CRC development}

To evaluate the contribution of CCR1 + hematopoietic myeloid cells in CRC progression, we next performed BM transfer experiments in which sub-lethally irradiated wild-type mice were reconstituted with BM from either wild-type or Ccr1-- mice of the C57BL/6 background (Fig. 3A), and then inoculated with CRC cells (MC38 and CMT93). Chimeras reconstituted with $\mathrm{Ccr} 1^{-/-}$BM exhibited depletion of circulating CCR1+ myeloid cells (Fig. 3B). IHC analysis indicated that CCR1+ myeloid cells accumulated around MC38 transplanted tumors and CMT93 liver metastases in recipient mice with wild-type $\mathrm{BM}$, whereas few $\mathrm{CCR} 1{ }^{+}$myeloid cells accumulated there in mice reconstituted with $\mathrm{Ccr1}^{-1-}$ BM (Fig. 3C, D). There were no significant differences in the distribution of circulating blood cells between recipient mice with wild-type BM and those with Ccr1/BM (Fig. 3E). We investigated the growth kinetics of MC38 transplanted tumors, and found that mice reconstituted with $\mathrm{Ccr}^{-1-}$ BM exhibited significantly smaller tumors compared with recipient mice of wild-type BM (Fig. 3F). On day 49 post-injection, the tumor size in the mice reconstituted with $\mathrm{Ccr1}^{-/-}$BM was $357 \pm 69 \mathrm{~mm}^{3}$, whereas that in the recipient mice with wild-type BM was $725 \pm 109 \mathrm{~mm}^{3}(P<0.05)$.

Next, we injected luc-expressing CMT93 cells into the spleen of the recipient mice reconstituted with either wild-type or $\mathrm{Ccr}^{-1-} \mathrm{BM}$ cells, and then monitored the metastasized cells within the liver by in vivo bioluminescence (Fig. 3G). After day 7 post-injection, mice reconstituted with $\mathrm{Ccr}^{-/-} \mathrm{BM}$ exhibited significantly lower numbers of 
metastasized cells compared with the control $(P<0.05)($ Fig. $3 \mathrm{H})$. We dissected the liver on day 21 post-injection, and found that macroscopic foci were observed in $56 \%$ (9 of 16) of mice reconstituted with $\mathrm{Ccr1}^{-/-}$BM, whereas observed in 100\% (15 of 15) of mice with wild-type BM ( $P<0.01$ : Supplementary Fig. S1C). To analyze the impact on the immune infiltrate composition, we quantified the density of $\mathrm{CD}^{+}$cytotoxic $\mathrm{T}$ cells, Foxp3 ${ }^{+}$ regulatory $\mathrm{T}$ (Treg) cells, CD31+ endothelial cells, and Ly6G+ neutrophils around tumors. In the MC38 transplanted tumors, depletion of CCR1 + hematopoietic cells led to a higher frequency of CD8 ${ }^{+} \mathrm{T}$ cells (mean, 12.6 vs. 16.0, $P<0.05$ ), whereas the numbers of Foxp3+ Treg cells, CD31+ cells, and Ly6G+ cells were decreased significantly (mean, 28.5 vs. $14.8, P<0.01 ; 28.9$ vs. $19.1, P<0.01$; and 5.7 vs. $3.7, P<0.05$, respectively) (Fig. 4A). Similarly, in the CMT93 liver metastases, lack of CCR1+ hematopoietic cells led to a higher frequency of CD8 ${ }^{+}$T cells (mean, 15.2 vs. 27.0; $P<0.01$ ), whereas the numbers of Foxp3+ Treg cells, CD31+ cells, and Ly6G+ cells were decreased significantly (mean, 30.3 vs. $17.4, P<0.01 ; 20.5$ vs. $17.6, P<0.05$; and 18.5 vs. $7.4, P<0.01$, respectively) (Fig. 4B). Thus, the concomitant increase in the number of CD8 ${ }^{+} \mathrm{T}$ cells, with the decrease in that of Foxp3 ${ }^{+}$Treg cells and CD31+endothelial cells, may indicate immune activation and diminished angiogenesis for mediating tumor regression in mice reconstituted with $\mathrm{Ccr}^{-/-}$hematopoietic cells.

Several studies have shown that MC38 cells are highly immunogenic with a high mutation load and are sensitive to anti-PD1 immune checkpoint inhibitors [23, 24]. Given depletion of CCR1 + hematopoietic myeloid cells suppressed tumor progression, we sought to test whether anti-PD1 therapy was more effective when the accumulation of CCR1 ${ }^{+}$cells was prohibited in subcutaneously growing MC38 tumors. As shown in Supplementary Fig. S2, we found that anti-PD1 mAb significantly decreased the growth of MC38 transplanted tumors in wild-type mice, and that the anti-tumor effect of anti-PD1 $\mathrm{mAb}$ was almost similar to the effect of genetic deletion of CCR1 in hematopoietic myeloid cells. However, no synergistic effects were observed by the combined inhibition of both PD1 and CCR1 pathways.

\subsection{The effect of a novel anti-CCR1 $\mathrm{mAb}$ in $C R C$ progression}

Although we previously used CCR1 antagonists (BL5923 and J-113863) for its suppressive effect in in vivo mouse models $[9,13]$, the dosage $(10-50 \mathrm{mg} / \mathrm{kg})$ was too 
high to be used in a clinical setting. In search for clinically applicable CCR1 inhibitors, we generated a novel and selective neutralizing mAb against CCR1, KM5908, to investigate the contribution of CCR1 in CRC progression. Flow cytometric analysis revealed that KM5908 specifically bound to human CCR1 as well as mouse CCR1 (Fig. 5A). In addition, a chemotaxis assay using CCR1-expressing THP-1 cells, a human monocytic leukemia cell line, indicated that KM5908 could efficiently inhibit the migratory response of THP-1 cells toward CCL15 in a dose-dependent manner: KM5908 at $3 \mu \mathrm{g} / \mathrm{mL}$ showed complete inhibition of CCL15-induced migration (Fig. 5B).

Finally, we evaluated KM5908 for its suppressive effects in the MC38 transplanted tumor model and CMT93 liver metastasis model to test whether targeting CCR1+ cells with anti-CCR1 mAb could potentially be a clinically applicable approach. We administered KM5908 or the isotype control mAb to wild-type mice injected with MC38 cells or CMT93 cells. In the MC38 transplanted tumor model, treatment with $10 \mu \mathrm{g} / \mathrm{g}$ of KM5908 significantly inhibited the growth of MC38 tumors compared with the isotype control (Fig. 5D). On day 46 post-injection, the tumor size in the KM5908-treated mice was $631 \pm 132 \mathrm{~mm}^{3}$, whereas that in the isotype-treated mice was $1556 \pm 351 \mathrm{~mm}^{3}(P<$ 0.05). In the CMT93 liver metastasis model, treatment with $10 \mu \mathrm{g} / \mathrm{g}$ of KM5908 significantly reduced the number of metastasized cells within the liver (Fig. 5E). On days 14 and 21 post-injection, there was a significant reduction of the liver luminescence in the KM5908-treated mice compared with the isotype-treated mice $(P<0.05)$. We dissected the liver on day 21 post-injection, and found that macroscopic foci were observed in 100\% (8 of 8 ) of the isotype-treated mice, whereas observed in only $44 \%$ (4 of 9 ) of the KM5908-treated mice ( $P<0.05$ : Supplementary Fig. S3). As anticipated, IHC analysis indicated that KM5908 treatment significantly reduced the number of accumulated CCR1 + cells around the MC38 tumors and CMT93 liver metastases compared with isotype treatment (mean, 1.2 vs. 3.2, $P<0.05$, and 4.8 vs. $9.0, P<0.05$, respectively) (Fig. 5C). Collectively, our data on preclinical models demonstrated that blockade of CCR1 signals by KM5908 elicited potent antitumor activity in CRC progression. 


\section{Discussion}

Accumulating evidence has revealed that the TME contains complex and multidirectional interactions between tumor cells and immune/non-immune stromal cells during tumor progression. Although infiltration of $\mathrm{CD}^{+} \mathrm{T}$ cells and cytotoxic $\mathrm{CD} 8^{+} \mathrm{T}$ cells is usually associated with a favorable prognosis of the cancer patients [1], the value of host-derived myeloid cells remains to be unraveled. Recently, there has been increasing interest in the role of myeloid cells; e.g., TAMs, TANs, and MDSCs. In experimental mouse models of cancer, it has been documented that myeloid cells can polarize into either anti- or pro-tumorigenic functional state $[17,19]$. The chemokine-chemokine receptor system is implicated in tumor progression by myeloid cell accumulation into the TME. The potential effects of CCR1+ myeloid cells have been shown in several types of cancer. We previously demonstrated that loss of SMAD4 from CRC cells causes recruitment of CCR1+ myeloid cells via the CCL15-CCR1 (human) or CCL9-CCR1 (mouse) axis to promote CRC invasion and metastasis [8-13]. Using a mouse model of liver metastasis, Rodero et al. reported that CCR1 expression in hematopoietic and stromal cells promoted tumor metastasis by myeloid cell infiltration and angiogenesis [16]. Liu et al. have recently reported that CCL15 is one of the most abundantly expressed chemokines in HCC with prognostic value, and that CCL15 recruits CCR $1^{+}$ monocytes to facilitate tumor growth and metastasis by inducing immune suppression and angiogenesis [14]. In a mouse model of breast cancer, Kitamura et al. reported that CCR2+ TAMs were recruited to lung metastases via CCL2-CCR2 axis, followed by recruitment of TAMs secreting CCL3 to enhance the retention of CCR1+TAMs within the lungs via CCL3-CCR1 axis, which resulted in extravasation of cancer cells [15]. In addition to CCR1, several types of chemokine receptors expressed on myeloid cells can facilitate tumor progression. In a mouse model of colitis-associated CRC, CXCR2 ligands were abundantly secreted from inflamed tumor cells, and accumulation of CXCR2+ MDSCs promoted colitis-induced tumor progression by inhibiting the cytotoxic activity of CD8 ${ }^{+} \mathrm{T}$ cells [25]. In a KRAS-mutant CRC mouse model, high expression of CXCL3 from CRC cells boosted CXCR2+ MDSC trafficking, and CXCR2 inhibition could overcome the resistance of anti-PD1 immunotherapy to KRAS-mutant CRC cells [26]. Recently, we have demonstrated that loss of SMAD4 from CRC cells upregulated expression of CXCL1 and CXCL8 to recruit CXCR2+ neutrophils, and that the recruited 
neutrophils, in turn, produced CXCL1 and CXCL8, which accelerated further accumulation of $\mathrm{CXCR}^{+}$neutrophils and shaped the cytokine/chemokine milieu of the TME [27].

CCR1 is expressed on peripheral blood monocytes, neutrophils, and macrophages as well as on immature myeloid cells and natural killer cells [28-30]. In the present study, we have shown that CCR1 is involved in the recruitment of myeloid cells, chiefly monocytes and neutrophils (Fig. 1), and that recruited CCR1+ cells can promote tumor progression by facilitating tumor angiogenesis (increase of intratumoral CD31+ endothelial cell) and immune suppression (decrease of CD8 ${ }^{+} \mathrm{T}$ cell and increase of Foxp3+ Treg cell) (Fig. 3, 4). Although neutrophils express higher levels of CCR1 compared with monocytes, the kinetics of CCR1 on cell surface recruitment in neutrophils may differ from that in monocytes. Expression of CCR1 in human neutrophils appears to be up-regulated by inflammatory cytokines, such as IFN- $\gamma$ and granulocyte-macrophage colony stimulating factor [31, 32]. Using clinical CRC samples, we have reported that most CCR1+ myeloid cells recruited to the primary and metastatic CRCs are granulocytic-MDSCs and TANs, respectively [11-13]. However, it remains to be determined whether MDSC and TAN are separate populations, because MDSC is a heterogeneous population of cells with immune suppression activity at various differentiation stages [33, 34]. Regarding the roles of CCR1 and CXCR2 expressed on neutrophils, Miyabe et al. recently reported that, in a mouse model of arthritis, CCR1 promoted neutrophil crawling on the endothelium, whereas CXCR2 amplified neutrophil retention and survival in the joint [35]. In HCC, CCL15 was reported to recruit CCR1+ monocytes to promote tumor growth and metastasis [14]. It appears that CCR1 ${ }^{+}$ hematopoietic myeloid cells are of diverse types, depending on the different TMEs. In addition to host immune cells, CCR1 is expressed in some types of tumor cells, including breast cancer, multiple myeloma, and hematolymphoid neoplasia [36, 37], which suggests that the mechanism mediating the pro-tumorigenic effects of CCR1 is a wide variety of mode.

Several lines of evidence support that CCR1 is also implicated in the pathogenesis of various diseases other than cancer [38-40]. For example, some CCR1 inhibitors have been used in phase I/II clinical trials for patients with rheumatoid arthritis, multiple sclerosis, and chronic obstructive pulmonary disease [41, 42]. Although clinical trials with 
CCR1 inhibitors have not shown promising results due to lack of enough efficacy [42, 43], CCR1 is still considered to be a therapeutic target [44]. On the other hand, drug repositioning is an attractive strategy because of the safety and cost [45]. In this preclinical study, we have demonstrated that murine CRC cell lines, MC38 and CMT93, induce the recruitment of CCR1 ${ }^{+}$myeloid cells to tumors, and that genetic CCR1 deficiency and/or pharmacological CCR1 blockade with anti-CCR1 mAb can exhibit robust anti-tumor effects on tumor growth and metastasis, which helps establish a preclinical rationale for CCR1 targeting in the treatment of CRC. To the best of our knowledge, the mAb described here is the first neutralizing Ab against CCR1 with high potency. Because there has been no clinically applicable therapeutics that block CCR1 in cancer therapy, its clinical application may provide a novel strategy targeting TME of CRC. 
Funding: This work was supported by grants from the Ministry of Education, Culture, Sports, Science and Technology of Japan, from Project Mirai Cancer Research, from The NOVARTIS Foundation (Japan) for the Promotion of Science, and from GAP Fund Program of Kyoto University (to K. Kawada). This work was also supported by the Kyowa Kirin Co., Ltd. (to K. Kawada, H. Hirai, and M. M. Taketo).

Author contributions: KK conceived the idea, designed the experiments, analyzed the data, and wrote the manuscript. YK designed the experiments, performed most experiments, analyzed the data, and wrote the manuscript. $\mathrm{HH}$ designed the experiments, performed some experiments, and analyzed the data. $\mathrm{RO}, \mathrm{KH}, \mathrm{HM}, \mathrm{GN}$, $\mathrm{TY}, \mathrm{RM}$, and $\mathrm{YI}$ assisted in performing the experiments. MK performed some experiments. MMK. designed the experiments, analyzed the data, and supervised the study. YS designed the experiments, and supervised the study. All authors discussed the results and commented the manuscript.

Potential Conflict of Interest: MK is an employee of Kyowa Kirin Co., Ltd. All other authors declare that they have no potential conflicts of interest.

Acknowledgments: The authors thank The Center for Anatomical, Pathological and Forensic Medical Research, Kyoto University Graduate School of Medicine, for preparing microscope slides. A part of this study was performed through the Joint Usage/ Research Center Program of the Radiation Biology Center, Kyoto University. Authors thank Naoya Fujita (Japanese Foundation For Cancer Research) for providing MC38. Authors also thank Yuji Mizuguchi, Akiko Furuya for monoclonal antibody production, and Keiko Suzuki for excellent technical assistance (Kyowa Kirin Co., Ltd.). 


\section{References}

1. F. Pages, B. Mlecnik, F. Marliot, G. Bindea, F.S. Ou, C. Bifulco, A. Lugli, I. Zlobec, T.T. Rau, M.D. Berger, I.D. Nagtegaal, E. Vink-Borger, A. Hartmann, C. Geppert, J. Kolwelter, S. Merkel, R. Grutzmann, M. Van den Eynde, A. Jouret-Mourin, A. Kartheuser, D. Leonard, C. Remue, J.Y. Wang, P. Bavi, M.H.A. Roehrl, P.S. Ohashi, L.T. Nguyen, S. Han, H.L. MacGregor, S. Hafezi-Bakhtiari, B.G. Wouters, G.V. Masucci, E.K. Andersson, E. Zavadova, M. Vocka, J. Spacek, L. Petruzelka, B. Konopasek, P. Dundr, H. Skalova, K. Nemejcova, G. Botti, F. Tatangelo, P. Delrio, G. Ciliberto, M. Maio, L. Laghi, F. Grizzi, T. Fredriksen, B. Buttard, M. Angelova, A. Vasaturo, P. Maby, S.E. Church, H.K. Angell, L. Lafontaine, D. Bruni, C. El Sissy, N. Haicheur, A. Kirilovsky, A. Berger, C. Lagorce, J.P. Meyers, C. Paustian, Z. Feng, C. Ballesteros-Merino, J. Dijkstra, C. van de Water, S. van Lent-van Vliet, N. Knijn, A.M. Musina, D.V. Scripcariu, B. Popivanova, M. Xu, T. Fujita, S. Hazama, N. Suzuki, H. Nagano, K. Okuno, T. Torigoe, N. Sato, T. Furuhata, I. Takemasa, K. Itoh, P.S. Patel, H.H. Vora, B. Shah, J.B. Patel, K.N. Rajvik, S.J. Pandya, S.N. Shukla, Y. Wang, G. Zhang, Y. Kawakami, F.M. Marincola, P.A. Ascierto, D.J. Sargent, B.A. Fox, J. Galon, International validation of the consensus Immunoscore for the classification of colon cancer: a prognostic and accuracy study, Lancet, 391 (2018) 2128-2139.

2. D.T. Le, J.N. Uram, H. Wang, B.R. Bartlett, H. Kemberling, A.D. Eyring, A.D. Skora, B.S. Luber, N.S. Azad, D. Laheru, B. Biedrzycki, R.C. Donehower, A. Zaheer, G.A. Fisher, T.S. Crocenzi, J.J. Lee, S.M. Duffy, R.M. Goldberg, A. de la Chapelle, M. Koshiji, F. Bhaijee, T. Huebner, R.H. Hruban, L.D. Wood, N. Cuka, D.M. Pardoll, N. Papadopoulos, K.W. Kinzler, S. Zhou, T.C. Cornish, J.M. Taube, R.A. Anders, J.R. Eshleman, B. Vogelstein, L.A. Diaz, Jr., PD-1 Blockade in Tumors with Mismatch-Repair Deficiency, N. Engl. J. Med., 372 (2015) 2509-2520.

3. D.I. Gabrilovich, S. Ostrand-Rosenberg, V. Bronte, Coordinated regulation of myeloid cells by tumours, Nat. Rev. Immunol., 12 (2012) 253-268.

4. C. Engblom, C. Pfirschke, M.J. Pittet, The role of myeloid cells in cancer therapies, Nat. Rev. Cancer, 16 (2016) 447-462.

5. D.F. Quail, J.A. Joyce, Microenvironmental regulation of tumor progression and 
metastasis, Nat. Med., 19 (2013) 1423-1437.

6. F. Balkwill, Cancer and the chemokine network, Nat. Rev. Cancer, 4 (2004) 540-550.

7. Y. Itatani, K. Kawada, S. Inamoto, T. Yamamoto, R. Ogawa, M.M. Taketo, Y. Sakai, The Role of Chemokines in Promoting Colorectal Cancer Invasion/Metastasis, Int. J. Mol. Sci., 17 (2016). E643

8. T. Kitamura, K. Kometani, H. Hashida, A. Matsunaga, H. Miyoshi, H. Hosogi, M. Aoki, M. Oshima, M. Hattori, A. Takabayashi, N. Minato, M.M. Taketo, SMAD4-deficient intestinal tumors recruit CCR1+ myeloid cells that promote invasion, Nat. Genet., 39 (2007) 467-475.

9. T. Kitamura, T. Fujishita, P. Loetscher, L. Revesz, H. Hashida, S. Kizaka-Kondoh, M. Aoki, M.M. Taketo, Inactivation of chemokine (C-C motif) receptor 1 (CCR1) suppresses colon cancer liver metastasis by blocking accumulation of immature myeloid cells in a mouse model, Proc. Natl. Acad. Sci. U. S. A., 107 (2010) 13063-13068.

10. H. Hirai, T. Fujishita, K. Kurimoto, H. Miyachi, S. Kitano, S. Inamoto, Y. Itatani, M. Saitou, T. Maekawa, M.M. Taketo, CCR1-mediated accumulation of myeloid cells in the liver microenvironment promoting mouse colon cancer metastasis, Clin. Exp. Metastasis, 31 (2014) 977-989.

11. Y. Itatani, K. Kawada, T. Fujishita, F. Kakizaki, H. Hirai, T. Matsumoto, M. Iwamoto, S. Inamoto, E. Hatano, S. Hasegawa, T. Maekawa, S. Uemoto, Y. Sakai, M.M. Taketo, Loss of SMAD4 from colorectal cancer cells promotes CCL15 expression to recruit CCR1+ myeloid cells and facilitate liver metastasis, Gastroenterology, 145 (2013) 1064-1075.

12. S. Inamoto, Y. Itatani, T. Yamamoto, S. Minamiguchi, H. Hirai, M. Iwamoto, S. Hasegawa, M.M. Taketo, Y. Sakai, K. Kawada, Loss of SMAD4 Promotes Colorectal Cancer Progression by Accumulation of Myeloid-Derived Suppressor Cells through the CCL15-CCR1 Chemokine Axis, Clin. Cancer Res., 22 (2016) 492-501.

13. T. Yamamoto, K. Kawada, Y. Itatani, S. Inamoto, R. Okamura, M. Iwamoto, E. Miyamoto, T.F. Chen-Yoshikawa, H. Hirai, S. Hasegawa, H. Date, M.M. Taketo, Y. Sakai, Loss of SMAD4 Promotes Lung Metastasis of Colorectal Cancer by 
Accumulation of CCR1+ Tumor-Associated Neutrophils through CCL15-CCR1 Axis, Clin. Cancer Res., 23 (2017) 833-844.

14. L.Z. Liu, Z. Zhang, B.H. Zheng, Y. Shi, M. Duan, L.J. Ma, Z.C. Wang, L.Q. Dong, P.P. Dong, J.Y. Shi, S. Zhang, Z.B. Ding, A.W. Ke, Y. Cao, X.M. Zhang, R. Xi, J. Zhou, J. Fan, X.Y. Wang, Q. Gao, CCL15 Recruits Suppressive Monocytes to Facilitate Immune Escape and Disease Progression in Hepatocellular Carcinoma, Hepatology, 69 (2019) 143-159.

15. T. Kitamura, B.Z. Qian, D. Soong, L. Cassetta, R. Noy, G. Sugano, Y. Kato, J. Li, J.W. Pollard, CCL2-induced chemokine cascade promotes breast cancer metastasis by enhancing retention of metastasis-associated macrophages, J. Exp. Med., 212 (2015) 1043-1059.

16. M.P. Rodero, C. Auvynet, L. Poupel, B. Combadiere, C. Combadiere, Control of both myeloid cell infiltration and angiogenesis by CCR1 promotes liver cancer metastasis development in mice, Neoplasia, 15 (2013) 641-648.

17. B.Z. Qian, J.W. Pollard, Macrophage diversity enhances tumor progression and metastasis, Cell, 141 (2010) 39-51.

18. Z.G. Fridlender, J. Sun, S. Kim, V. Kapoor, G. Cheng, L. Ling, G.S. Worthen, S.M. Albelda, Polarization of tumor-associated neutrophil phenotype by TGF-beta: "N1" versus "N2" TAN, Cancer Cell, 16 (2009) 183-194.

19. A. Mantovani, M.A. Cassatella, C. Costantini, S. Jaillon, Neutrophils in the activation and regulation of innate and adaptive immunity, Nat. Rev. Immunol., 11 (2011) 519-531.

20. WO2013/005649.

21. T. Yoshii, R. Kono, Establishement of a rabbit lung cell line (RL-33) and its viral susceptibility, Jpn. J. Med. Sci. Biol., 30 (1977) 149-157.

22. J.L. Gao, T.A. Wynn, Y. Chang, E.J. Lee, H.E. Broxmeyer, S. Cooper, H.L. Tiffany, H. Westphal, J. Kwon-Chung, P.M. Murphy, Impaired host defense, hematopoiesis, granulomatous inflammation and type 1-type 2 cytokine balance in mice lacking CC chemokine receptor 1, J. Exp. Med., 185 (1997) 1959-1968.

23. M. Efremova, D. Rieder, V. Klepsch, P. Charoentong, F. Finotello, H. Hackl, N. Hermann-Kleiter, M. Lower, G. Baier, A. Krogsdam, Z. Trajanoski, Targeting immune checkpoints potentiates immunoediting and changes the dynamics of 
tumor evolution, Nat Commun, 9 (2018) 32.

24. B. Gong, K. Kiyotani, S. Sakata, S. Nagano, S. Kumehara, S. Baba, B. Besse, N. Yanagitani, L. Friboulet, M. Nishio, K. Takeuchi, H. Kawamoto, N. Fujita, R. Katayama, Secreted PD-L1 variants mediate resistance to PD-L1 blockade therapy in non-small cell lung cancer, J. Exp. Med., 216 (2019) 982-1000.

25. H. Katoh, D. Wang, T. Daikoku, H. Sun, S.K. Dey, R.N. Dubois, CXCR2-expressing myeloid-derived suppressor cells are essential to promote colitis-associated tumorigenesis, Cancer Cell, 24 (2013) 631-644.

26. W. Liao, M.J. Overman, A.T. Boutin, X. Shang, D. Zhao, P. Dey, J. Li, G. Wang, Z. Lan, J. Li, M. Tang, S. Jiang, X. Ma, P. Chen, R. Katkhuda, K. Korphaisarn, D. Chakravarti, A. Chang, D.J. Spring, Q. Chang, J. Zhang, D.M. Maru, D.Y. Maeda, J.A. Zebala, S. Kopetz, Y.A. Wang, R.A. DePinho, KRAS-IRF2 Axis Drives Immune Suppression and Immune Therapy Resistance in Colorectal Cancer, Cancer Cell, 35 (2019) 559-572.

27. R. Ogawa, T. Yamamoto, H. Hirai, K. Hanada, Y. Kiyasu, G. Nishikawa, R. Mizuno, S. Inamoto, Y. Itatani, Y. Sakai, K. Kawada, Loss of SMAD4 Promotes Colorectal Cancer Progression by Recruiting Tumor-Associated Neutrophils via the CXCL1/8-CXCR2 Axis, Clin. Cancer Res., 25 (2019) 2887-2899.

28. L. Patel, S.J. Charlton, J.K. Chambers, C.H. Macphee, Expression and functional analysis of chemokine receptors in human peripheral blood leukocyte populations, Cytokine, 14 (2001) 27-36.

29. J.L. Gao, D.B. Kuhns, H.L. Tiffany, D. McDermott, X. Li, U. Francke, P.M. Murphy, Structure and functional expression of the human macrophage inflammatory protein 1 alpha/RANTES receptor, J. Exp. Med., 177 (1993) 1421-1427.

30. A. Mantovani, R. Bonecchi, M. Locati, Tuning inflammation and immunity by chemokine sequestration: decoys and more, Nat. Rev. Immunol., 6 (2006) 907-918.

31. S.S. Cheng, J.J. Lai, N.W. Lukacs, S.L. Kunkel, Granulocyte-macrophage colony stimulating factor up-regulates CCR1 in human neutrophils, J. Immunol., 166 (2001) 1178-1184.

32. R. Bonecchi, N. Polentarutti, W. Luini, A. Borsatti, S. Bernasconi, M. Locati, C. Power, A. Proudfoot, T.N. Wells, C. Mackay, A. Mantovani, S. Sozzani, 
Up-regulation of CCR1 and CCR3 and induction of chemotaxis to CC chemokines by IFN-gamma in human neutrophils, J. Immunol., 162 (1999) 474-479.

33. S.B. Coffelt, M.D. Wellenstein, K.E. de Visser, Neutrophils in cancer: neutral no more, Nat. Rev. Cancer, 16 (2016) 431-446.

34. V. Bronte, S. Brandau, S.H. Chen, M.P. Colombo, A.B. Frey, T.F. Greten, S. Mandruzzato, P.J. Murray, A. Ochoa, S. Ostrand-Rosenberg, P.C. Rodriguez, A. Sica, V. Umansky, R.H. Vonderheide, D.I. Gabrilovich, Recommendations for myeloid-derived suppressor cell nomenclature and characterization standards, Nat Commun, 7 (2016) 12150.

35. Y. Miyabe, C. Miyabe, T.T. Murooka, E.Y. Kim, G.A. Newton, N.D. Kim, B. Haribabu, F.W. Luscinskas, T.R. Mempel, A.D. Luster, Complement C5a Receptor is the Key Initiator of Neutrophil Adhesion Igniting Immune Complex-induced Arthritis, Sci Immunol, 2 (2017) eaaj2195.

36. S.Y. Shin, D.H. Lee, J. Lee, C. Choi, J.Y. Kim, J.S. Nam, Y. Lim, Y.H. Lee, C-C motif chemokine receptor 1 (CCR1) is a target of the EGF-AKT-mTOR-STAT3 signaling axis in breast cancer cells, Oncotarget, 8 (2017) 94591-94605.

37. K. Vandyke, M.N. Zeissig, D.R. Hewett, S.K. Martin, K.M. Mrozik, C.M. Cheong, P. Diamond, L.B. To, S. Gronthos, D.J. Peet, P.I. Croucher, A.C.W. Zannettino, HIF-2alpha Promotes Dissemination of Plasma Cells in Multiple Myeloma by Regulating CXCL12/CXCR4 and CCR1, Cancer Res., 77 (2017) 5452-5463.

38. P.P. Tak, A. Balanescu, V. Tseluyko, S. Bojin, E. Drescher, D. Dairaghi, S. Miao, V. Marchesin, J. Jaen, T.J. Schall, P. Bekker, Chemokine receptor CCR1 antagonist CCX354-C treatment for rheumatoid arthritis: CARAT-2, a randomised, placebo controlled clinical trial, Ann. Rheum. Dis., 72 (2013) 337-344.

39. A.R. Karash, A. Gilchrist, Therapeutic potential of CCR1 antagonists for multiple myeloma, Future Med. Chem., 3 (2011) 1889-1908.

40. V. Ninichuk, H.J. Anders, Chemokine receptor CCR1: a new target for progressive kidney disease, Am. J. Nephrol., 25 (2005) 365-372.

41. R.P. Gladue, M.F. Brown, S.H. Zwillich, CCR1 antagonists: what have we learned from clinical trials, Curr. Top. Med. Chem., 10 (2010) 1268-1277.

42. Z. Szekanecz, A.E. Koch, Successes and failures of chemokine-pathway targeting in rheumatoid arthritis, Nat. Rev. Rheumatol., 12 (2016) 5-13. 
43. J. Pease, R. Horuk, Chemokine receptor antagonists, J. Med. Chem., 55 (2012) 9363-9392.

44. R.P. Gladue, S.H. Zwillich, A.T. Clucas, M.F. Brown, CCR1 antagonists for the treatment of autoimmune diseases, Curr Opin Investig Drugs, 5 (2004) 499-504.

45. F. Bertolini, V.P. Sukhatme, G. Bouche, Drug repurposing in oncology--patient and health systems opportunities, Nat. Rev. Clin. Oncol., 12 (2015) 732-742. 


\section{Figure legends}

Fig. 1. Accumulation of CCR1+ myeloid cells around CRC tumors.

A, Flow cytometric analysis to investigate CCR1 expression of neutrophils (left), monocytes (middle), and lymphocytes (right) in peripheral blood and BM. The solid lines show anti-CCR1, while dotted lines show isotype control. B, qRT-PCR analysis for Ccr1 mRNA. C, Expression of mRNAs for CCR1 ligands determined by RT-PCR. p.c. indicates positive control (spleen for CCL3, 4, 5, 6, and 9; colon for CCL7), while n.c. indicates negative control (distilled water instead of RNA). D, IHC analysis of MC38 subcutaneous transplanted tumors. Scale bar, $100 \mu \mathrm{m}$. E, IHC analysis of CMT93 liver metastatic tumors. Scale bar, $100 \mu \mathrm{m}$. F, Simultaneous immunofluorescence staining of MC38 subcutaneous transplanted tumors for CCR1 (red) and MMP2, MMP9, iNOS, or VEGF (green). Scale bar, $20 \mu \mathrm{m}$. G, Simultaneous immunofluorescence staining of CMT93 liver metastatic tumors for CCR1 (red) and MMP2, MMP9, iNOS, or VEGF (green). Scale bar, $20 \mu \mathrm{m}$.

Fig. 2. Lack of $C C R 1$ in host mice suppresses $C R C$ tumor growth and metastasis. A, Representative macroscopic views of the MC38 subcutaneous transplanted tumors dissected from the wild-type mice and $\mathrm{Ccr}^{1--}$ mice. Scale bar, $10 \mathrm{~mm}$. B, Growth curves of the MC38 subcutaneous transplanted tumors in the wild-type mice and $\mathrm{CCr}^{1-\text { - }}$ mice. Mean; bar, \pm SEM, $n=8-13$ mice in each group. ${ }^{*} P<0.05$ by Student's $t$-test. C, Representative in vivo bioluminescence images of CMT93-luc liver metastases in the wild-type mice and $\mathrm{CCr}^{1-/}$ mice. D, Quantification of CMT93 liver metastatic lesions (photon counts) in wild-type mice and Ccr ${ }^{-/}$mice. Mean; bar, \pm SEM, $n=9-12$ mice in each group. ${ }^{*} P<0.05$ by Mann-Whitney $U$ test.

Fig. 3. Lack of CCR1 in hematopoietic myeloid cells suppresses CRC tumor growth and metastasis.

A, Schema of BM transfer experiments. B, PCR analysis of wild-type (733 kb) and mutated allele $(1481 \mathrm{~kb})$ of CCR1. Genotypic analysis of tail DNA derived from heterozygous crosses (left). Wild-type hosts were sublethally irradiated and given with BM from either $\mathrm{Ccr}^{-1-}$ mice (middle) or wild-type mice (right). p.c., positive control. n.c., negative control. C, IHC analysis of MC38 subcutaneous transplanted tumors. Scale bar, 
$100 \mu \mathrm{m}$. D, IHC analysis of CMT93 liver metastatic tumors. Scale bar, $100 \mu \mathrm{m}$. E, Distribution of total leukocytes in peripheral blood. $n=4-5$ mice in each group. $\mathbf{F}$, Growth curves of the MC38 subcutaneous transplanted tumors in wild-type recipient hosts reconstituted with wild-type BM and Ccr1-/- BM. Mean; bar, \pm SEM, $n=8-11$ mice in each group. * $P<0.05$ by Student's $t$-test. $\mathbf{G}$, Representative in vivo bioluminescence images of CMT93-luc liver metastases in wild-type recipient hosts reconstituted with wild-type BM and Ccr1-/- BM. H, Quantification of CMT93 liver metastatic lesions (photon counts) in wild-type recipient hosts reconstituted with wild-type BM and Ccr1/- BM. Mean; bar, \pm SEM, $n=15-16$ mice in each group. ${ }^{*} P<0.05$ by Mann-Whitney $U$ test.

Fig. 4. Lack of CCR1 in hematopoietic myeloid cells changes the immune infiltrate composition of TME.

A, In the MC38 subcutaneous transplanted tumors, wild-type recipient host reconstituted with $\mathrm{Ccr1}^{-1-} \mathrm{BM}$ exhibited higher frequency of $\mathrm{CD}^{+} \mathrm{T}$ cell accumulation, while Foxp3 ${ }^{+}$ Treg cell infiltration and CD31+ microvessel density within tumors were decreased. Scale bar, $100 \mu \mathrm{m}$. Mean; bar, \pm SEM, $n=4-5$ mice in each group. ${ }^{*} P<0.05$ and ${ }^{\star \star} P<0.01$ by Student's $t$-test. B, In the CMT93 liver metastases, wild-type recipient host reconstituted with $\mathrm{Ccr1}^{-/-} \mathrm{BM}$ exhibited higher frequency of $\mathrm{CD}^{+} \mathrm{T}$ cell accumulation, while Foxp3+ Treg cell infiltration and CD31+ microvessel density within tumors were decreased. Scale bar, $100 \mu \mathrm{m}$. Mean; bar, \pm SEM, $n=4-5$ mice in each group. ${ }^{*} P<0.01$ by Student's t-test.

Fig. 5. A novel anti-CCR1 mAb, KM5908, suppresses CRC tumor growth.

A, Flowcytometric binding assay of KM5908 to CCR1-expressing cell lines. Tinted, dashed and solid lines indicate $0,0.01$ and $0.1 \mathrm{mg} / \mathrm{mL}$ of antibody respectively. KM5908 specifically recognized both human and mouse CCR1. B, Chemotaxis assay of THP-1 cells to CCL15. Y-axis indicates relative number of the cells migrated to the lower chamber. KM5908 inhibited chemotaxis of THP-1 in a dose dependent manner. C, Accumulation of $\mathrm{CCR} 1^{+}$cells around the MC38 subcutaneous transplanted tumors (left) and CMT93 liver metastatic tumors (right). Mean; bar, \pm SEM, $n=3-4$ mice in each group and $n=10-20$ views in each mouse. ${ }^{\star} P<0.05$ by Student's $t$-test. D, Growth curves of the MC38 subcutaneous transplanted tumors in wild-type mice treated with KM5908 and 
isotype control. Mean; bar, \pm SEM, $n=10$ mice in each group. ${ }^{*} P<0.05$ by Student's $t$-test. Schema of KM5908-treated experiment was shown (top). E, Quantification of CMT93 liver metastatic lesions (photon counts) in wild-type mice treated with KM5908 and isotype control. Mean; bar, \pm SEM, $n=8-9$ mice in each group. ${ }^{*} P<0.05$ by Mann-Whitney $U$ test. Schema of KM5908-treated experiment was shown (top). 


\section{Supplementary figure legends}

Supplementary Figure S1. Tumor progression in wild-type mice and $\mathrm{CCr}^{-1-}$ mice. A, Distribution of total leukocytes in peripheral blood. $n=4-5$ mice in each group. B, Representative macroscopic views of the liver dissected from wild-type and $\mathrm{Ccr}^{1-1-}$ mice injected with CMT93-luc cells. Scale bar, $10 \mathrm{~mm}$. C, Representative macroscopic views of the liver dissected from mice reconstituted with $\mathrm{Ccr}^{1-1-} \mathrm{BM}$ and wild-type BM injected with CMT93-luc cells. Scale bar, $10 \mathrm{~mm}$.

Supplementary Figure S2. Anti-tumor effect of anti-PD1 therapy in CCR1 deletion of hematopoietic myeloid cells.

Twelve weeks after BM transplantation, recipient mice were subcutaneously injected with MC38 cells. On day 7 post-injection, tumor-bearing mice were randomly divided into the four groups: recipient mice of wild-type BM or $\mathrm{Ccr}^{-1-} \mathrm{BM}$ treated with anti-PD1 mAb or isotype control. Anti-PD1 mAb or isotype control was injected intraperitoneally on days $7,11,14,18$. A, Representative macroscopic views of the MC38 subcutaneous transplanted tumors dissected from recipient mice of wild-type BM or $\mathrm{Ccr}^{-1-\mathrm{BM}}$, treated with anti-PD1 mAb or isotype control. Scale bar, $10 \mathrm{~mm}$. B, Growth curves of the MC38 subcutaneous transplanted tumors in recipient mice of wild-type BM or Ccr1 ${ }^{-1-} \mathrm{BM}$, treated with anti-PD1 mAb or isotype control. Mean; bar, \pm SEM, $n=6$ mice in each group. ${ }^{*} P<0.05$ by Student's $t$-test.

Supplementary Figure S3. Anti-tumor effect of a novel anti-CCR1 mAb, KM5908. Representative macroscopic views of the liver dissected from mice treated with KM5908 and isotype control. Scale bar, $10 \mathrm{~mm}$. 


\section{Supplementary Table S1}

List of Antibodies used for IHC and IFC.

\begin{tabular}{|l|l|l|l|l|}
\hline Antibody & Host & Company & Dilution (IHC) & Dilution (IF) \\
\hline anti-mCCR1 & rabbit & made in our lab ${ }^{13}$ & $1: 500$ & $1: 500$ \\
\hline anti-mCCL9 & goat & R\&D Systems & $1: 50$ & \\
\hline anti-mLy6G & rat & BioXcell & $1: 1000$ & \\
\hline anti-mCD8 & rat & eBiosciene & $1: 100$ & \\
\hline anti-mFOXP3 & rabbit & Cell Signaling & $1: 100$ & \\
\hline anti-mCD31 & rat & Cell Signaling & $1: 100$ & \\
\hline anti-mMMP2 & goat & R\&D Systems & & $1: 20$ \\
\hline anti-mMMP9 & goat & R\&D Systems & & $1: 20$ \\
\hline anti-mVEGF & goat & R\&D Systems & & $1: 200$ \\
\hline anti-miNOS & rat & BioLegend & & $1: 20$ \\
\hline
\end{tabular}




\section{Supplementary Table S2}

List of Antibodies used for FACS.

\begin{tabular}{|l|l|l|l|}
\hline Antibody & Host & Conjugate & Company \\
\hline anti-CD11b & rat & PE-Cy7 & BD Pharmingen \\
\hline anti-Ly6G & rat & FITC & BD Pharmingen \\
\hline anti-CD115 & rat & PE & BioLegend \\
\hline Anti-CCR1 & mouse & Alexa Fluor 647 & Kyowa Kirin Co., Ltd. \\
\hline
\end{tabular}




\section{Fig.1}

A



D

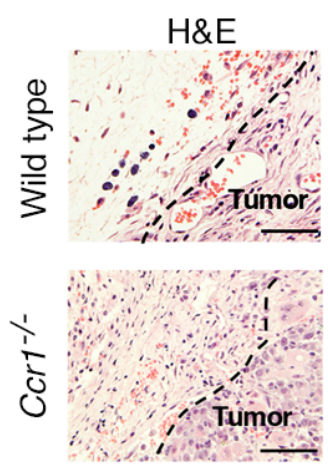

$\mathbf{F}$



MMP9
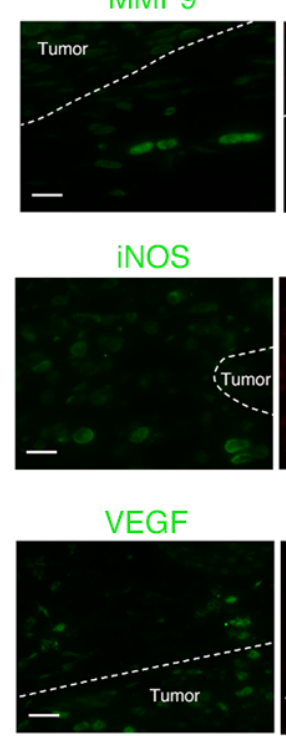

CCR1

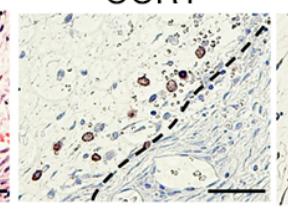

CCL9
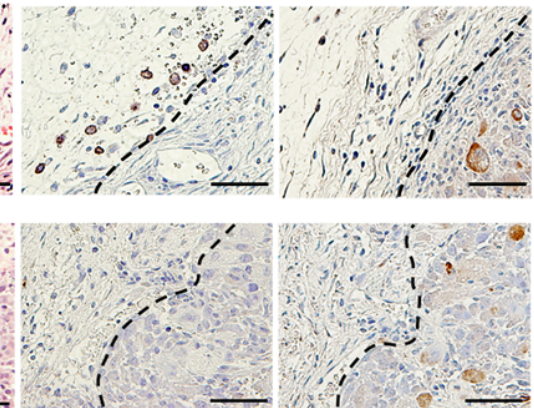

CCR1 MMP2/CCR1/DAPI
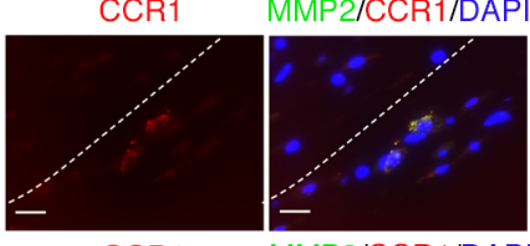

CCR1
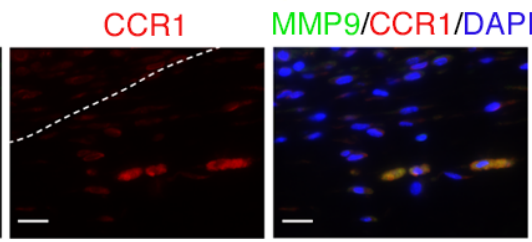

CCR1
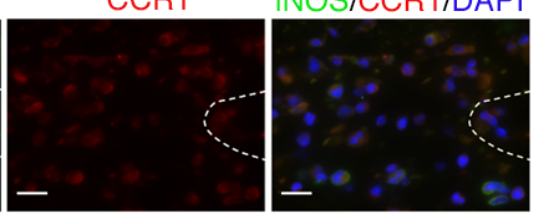

CCR1
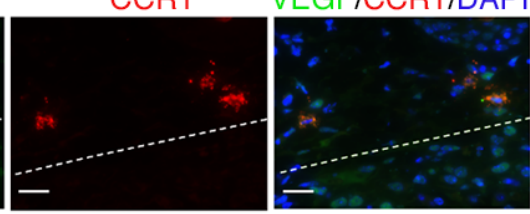

NOS/CCR1/DAPI
B

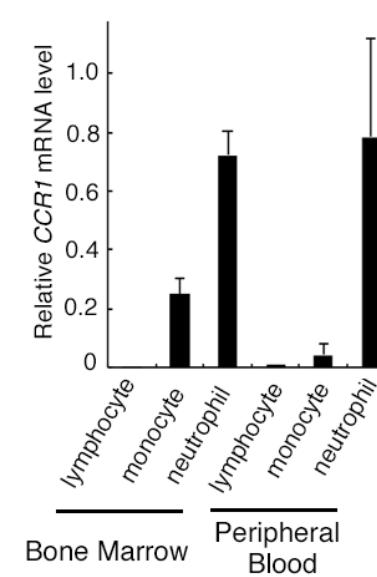

E
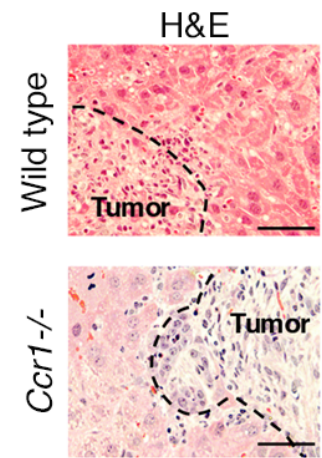

G

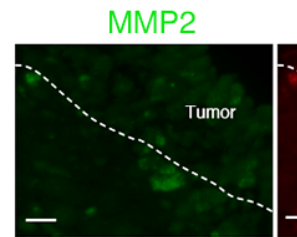

MMP9
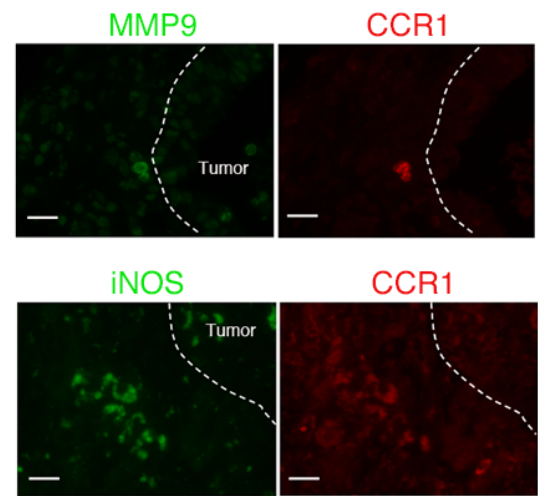

CCR1

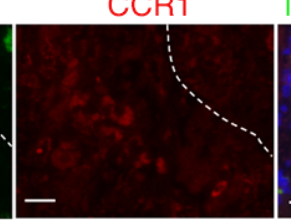

VEGF

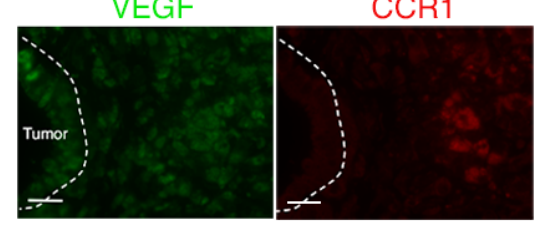

iNOS/CCR1/DAPI

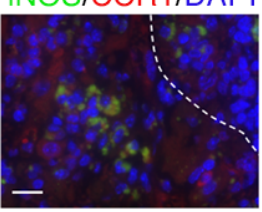

MMP2/CCR1/DAPI

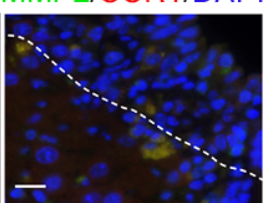

MMP9/CCR1/DAPI

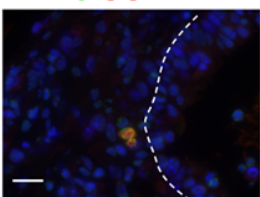

VEGF/CCR1/DAPI

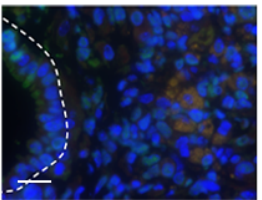




\section{Fig.2}

A

$\frac{9}{2}$

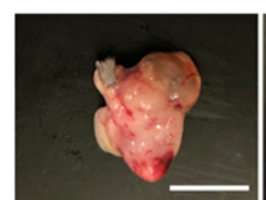

$\frac{1}{0}$

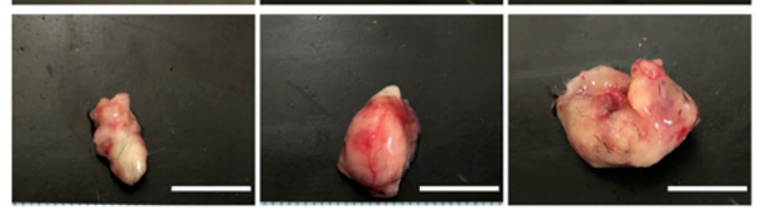

B

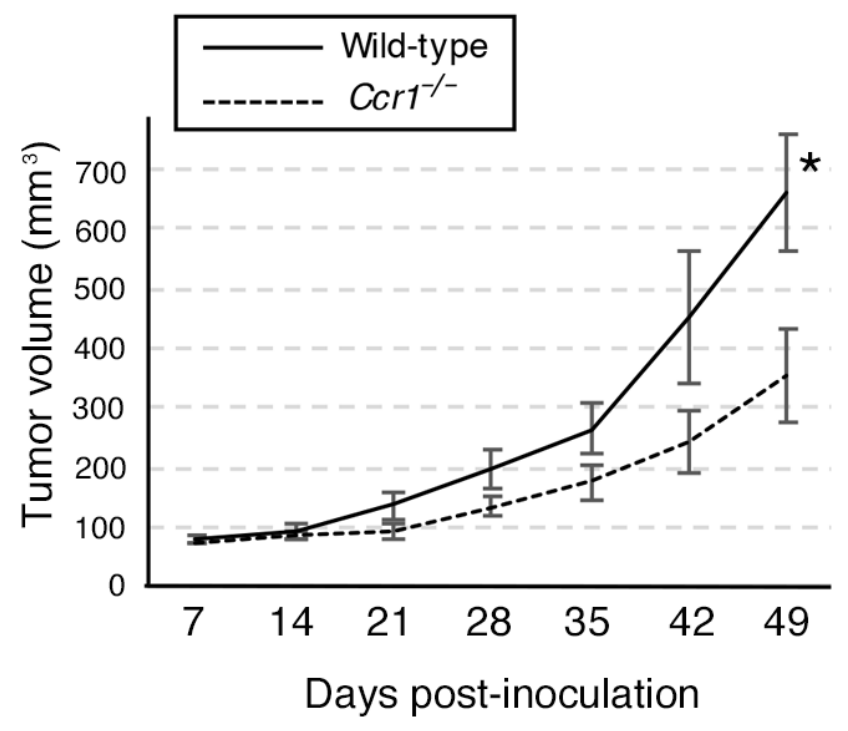

D

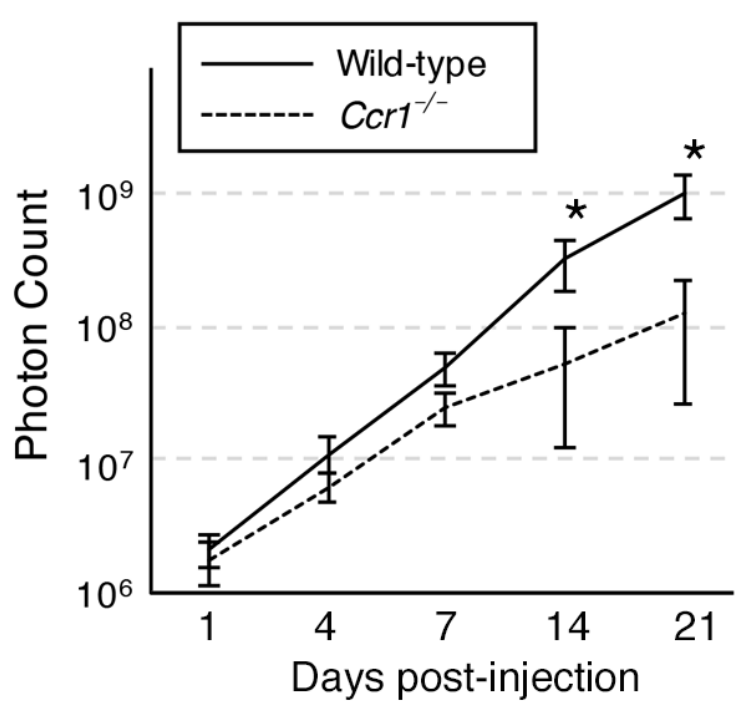

Days post-inoculation

Days post-injection
C

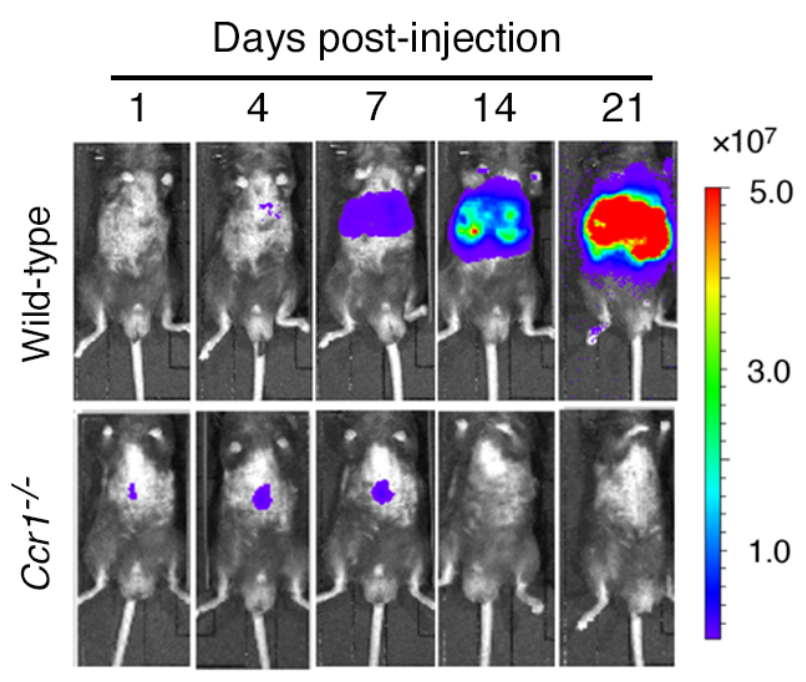




\section{Fig.3}

A

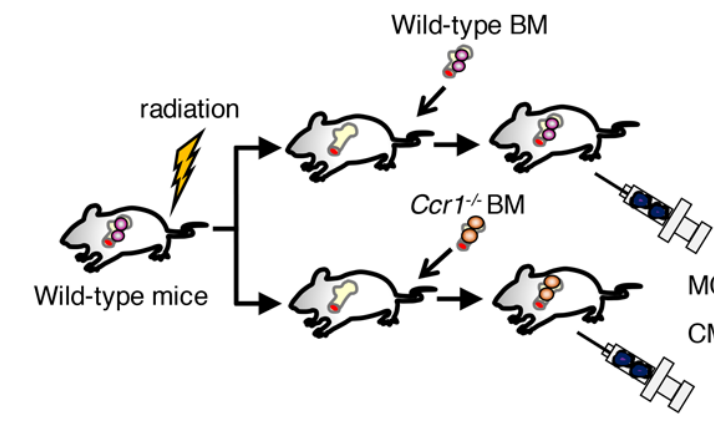

C

$H \& E$

CCR1

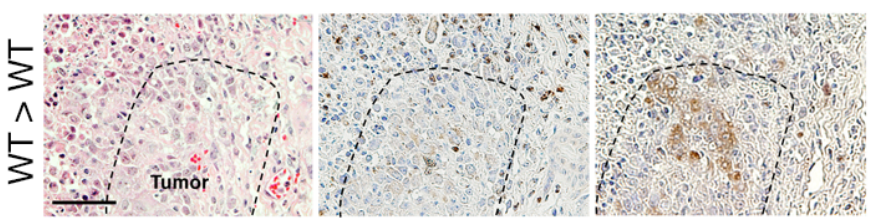

々

$\frac{1}{1}$

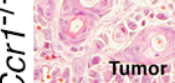

E

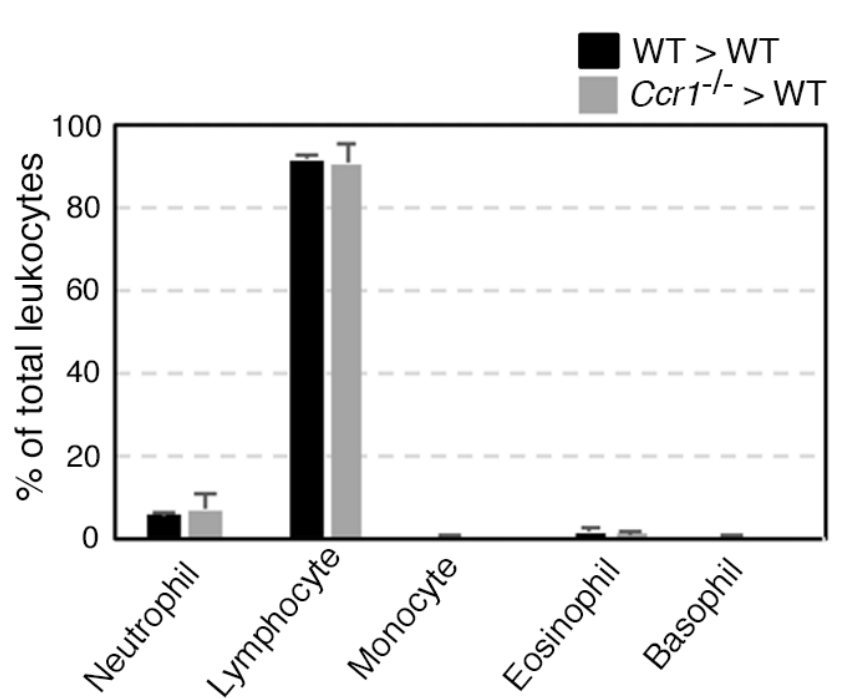

G

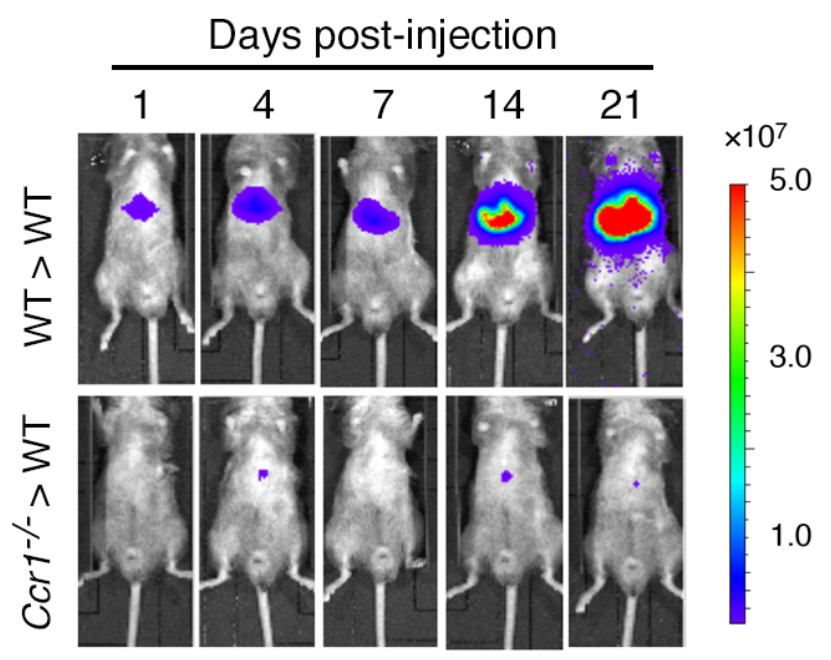

B

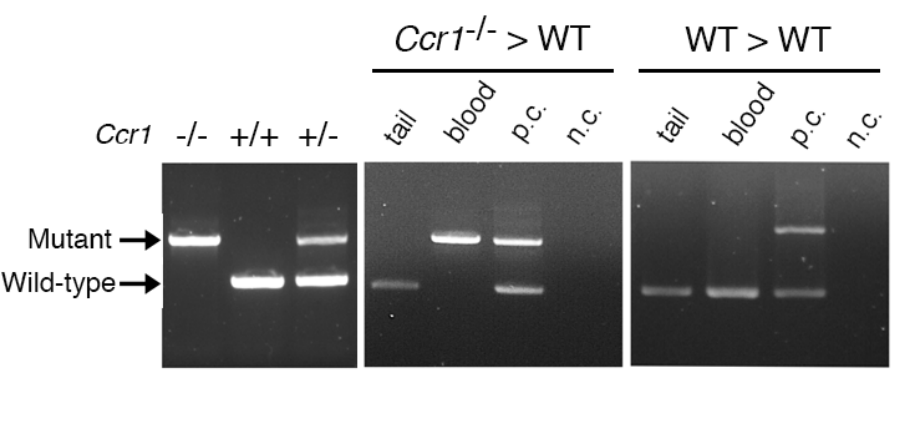

D



CCR1

CCL9

$\mathbf{F}$

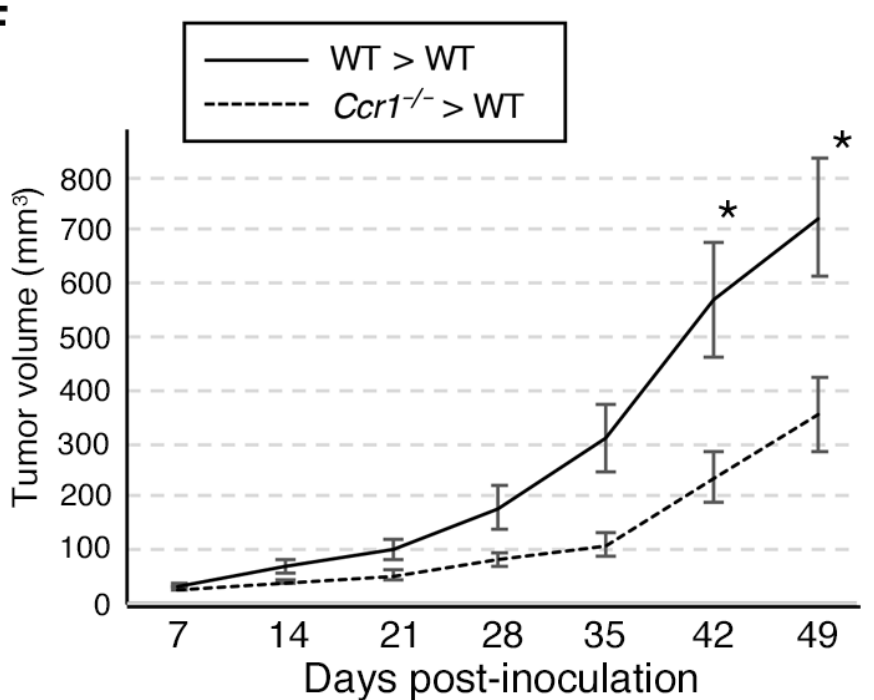

H

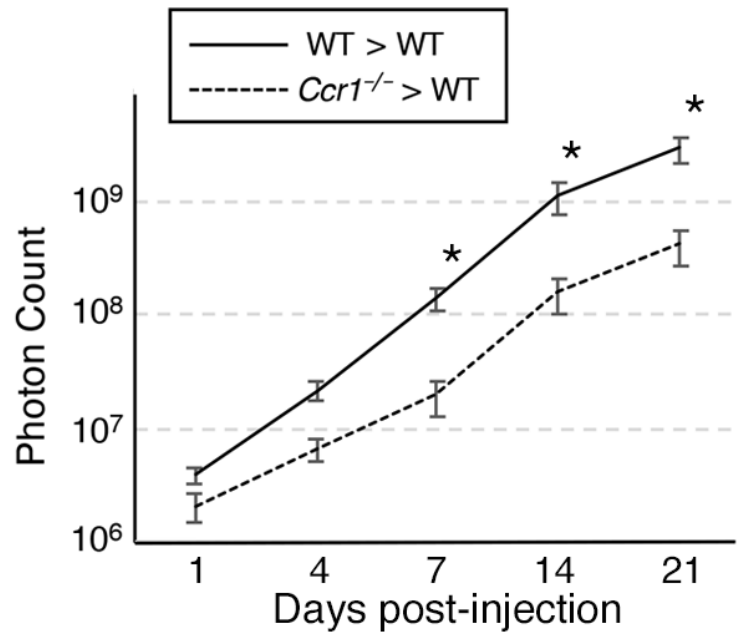


Fig.4

A

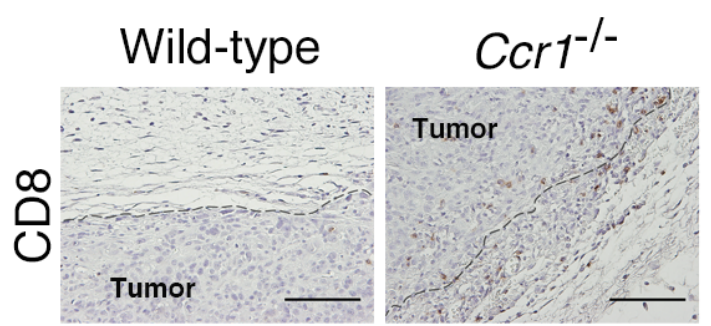

$m$
$\stackrel{2}{x}$
ํ.
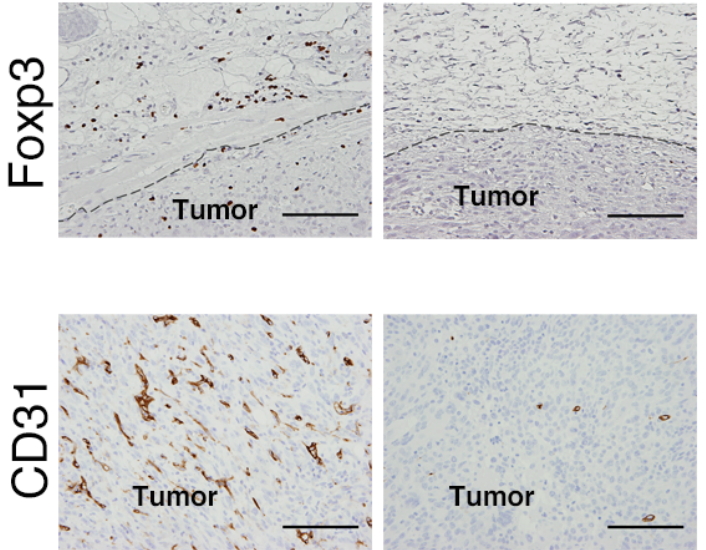

Tumor

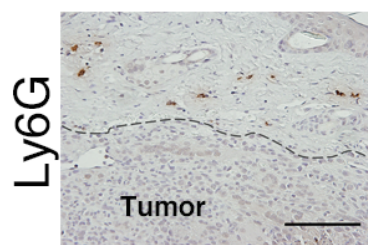

Tumor
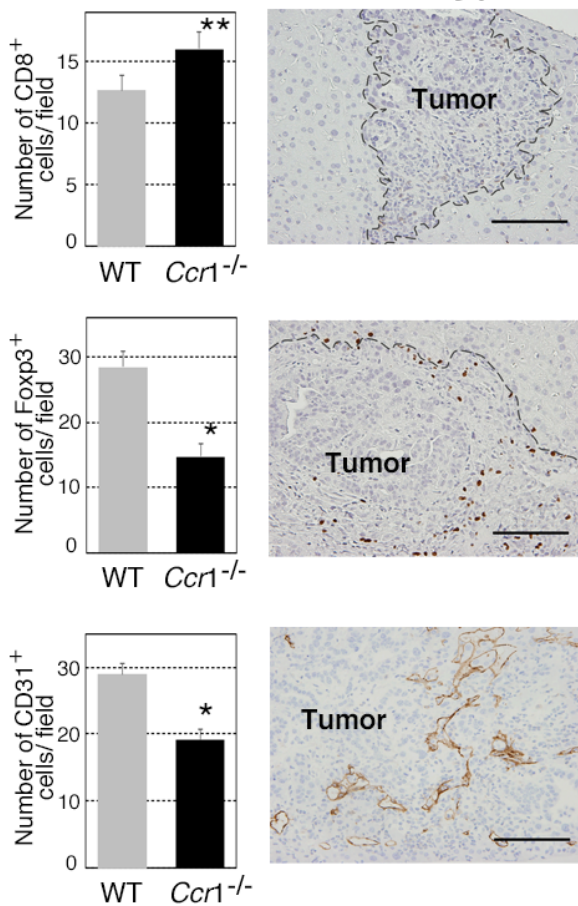

B
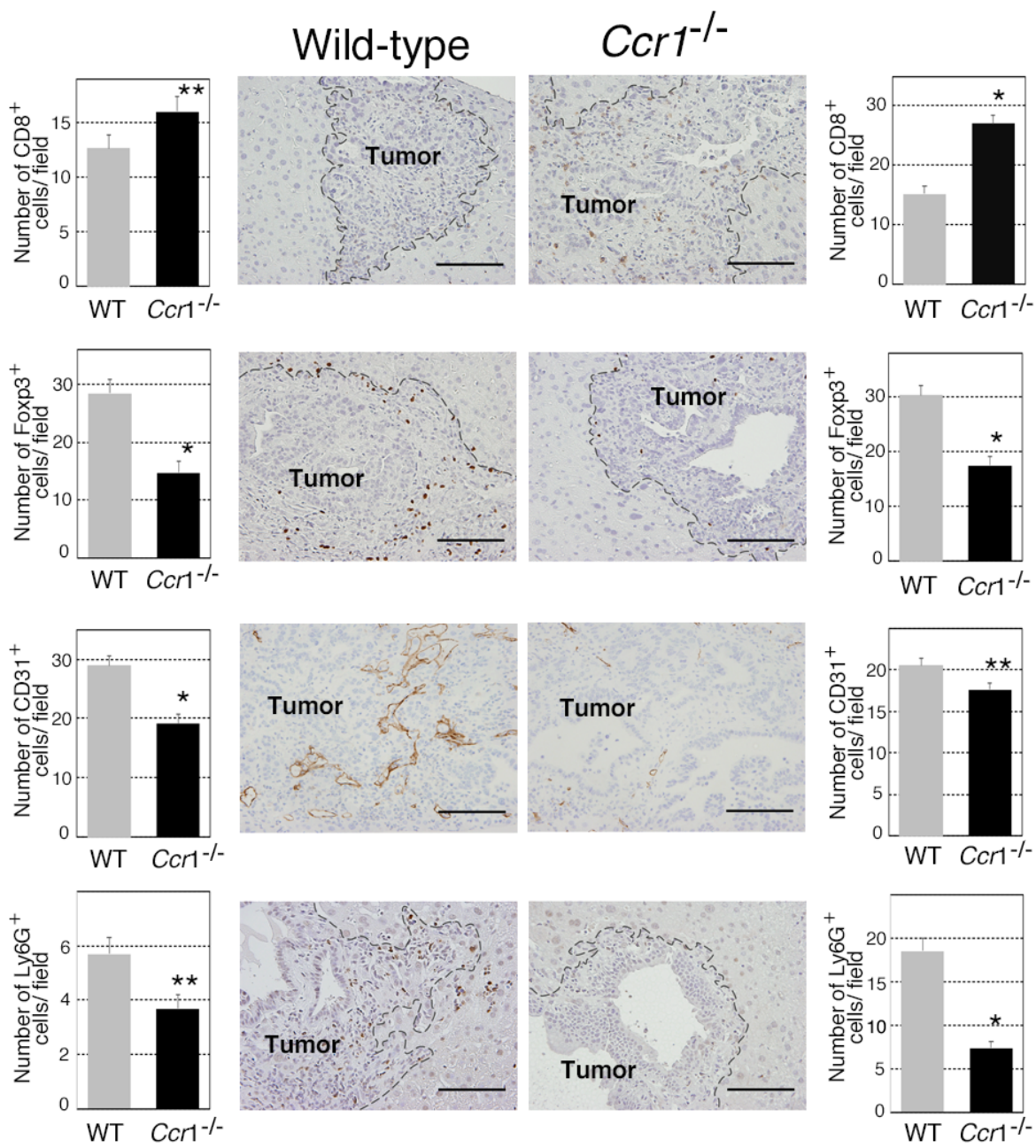
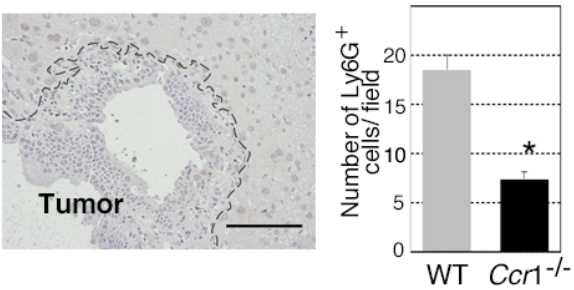
Fig.5

A
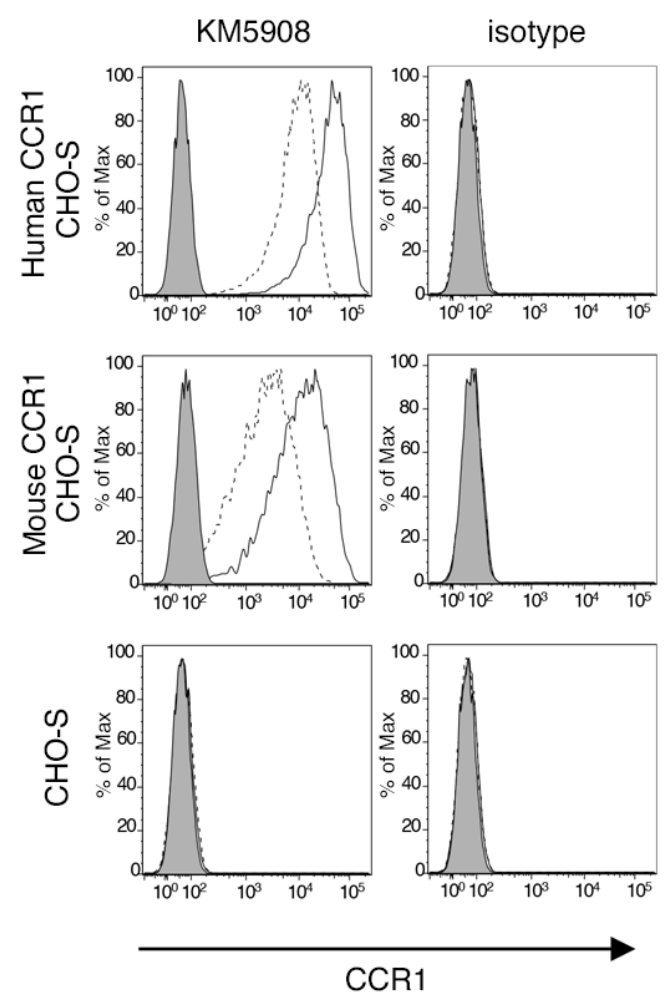

D
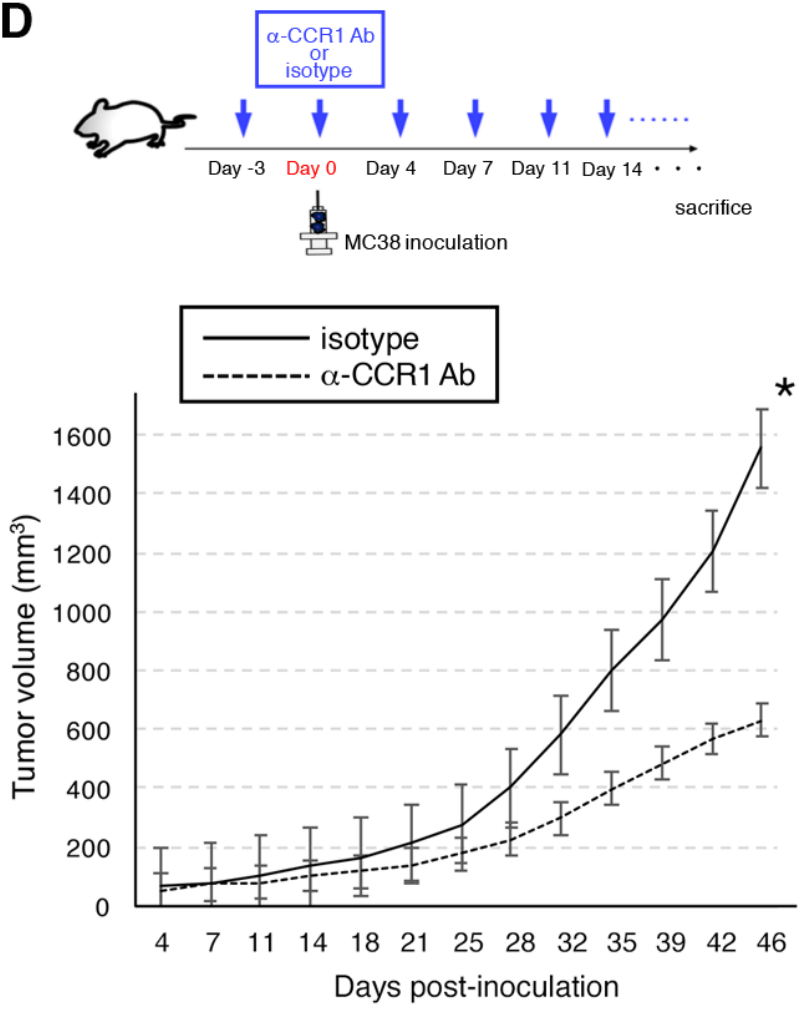

B

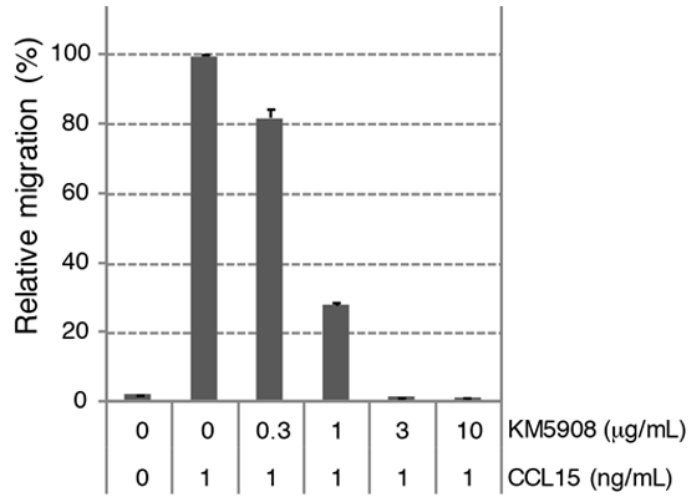

C



E
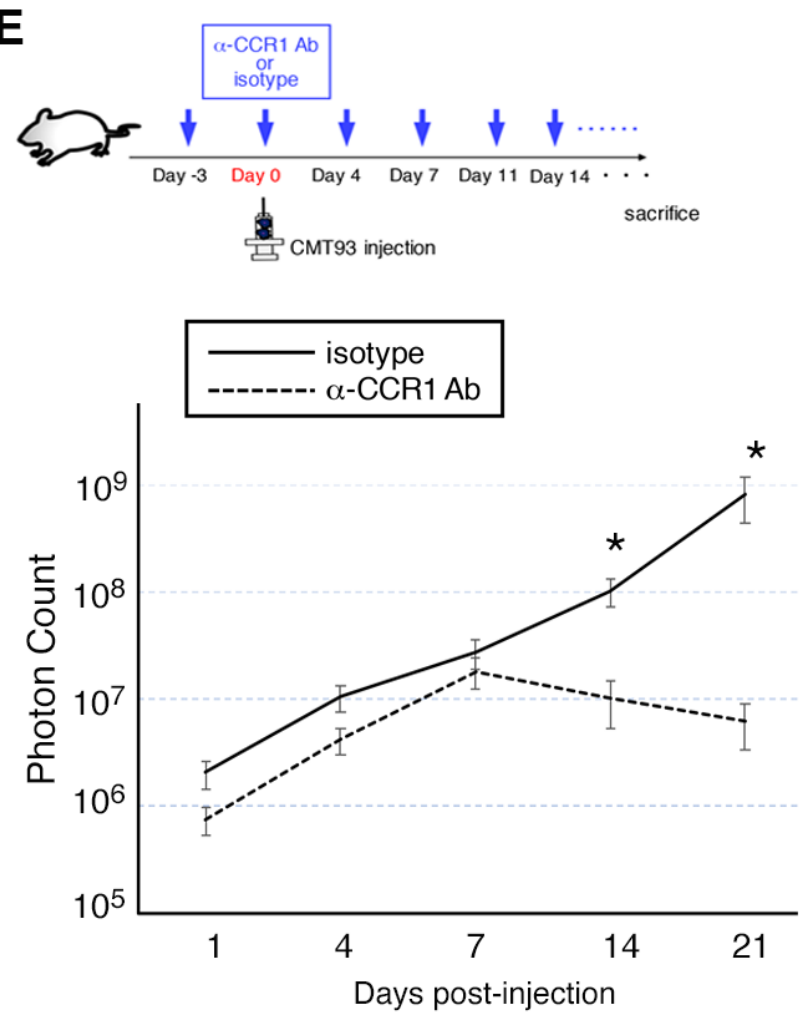


\section{Supplementary Fig.1}

A

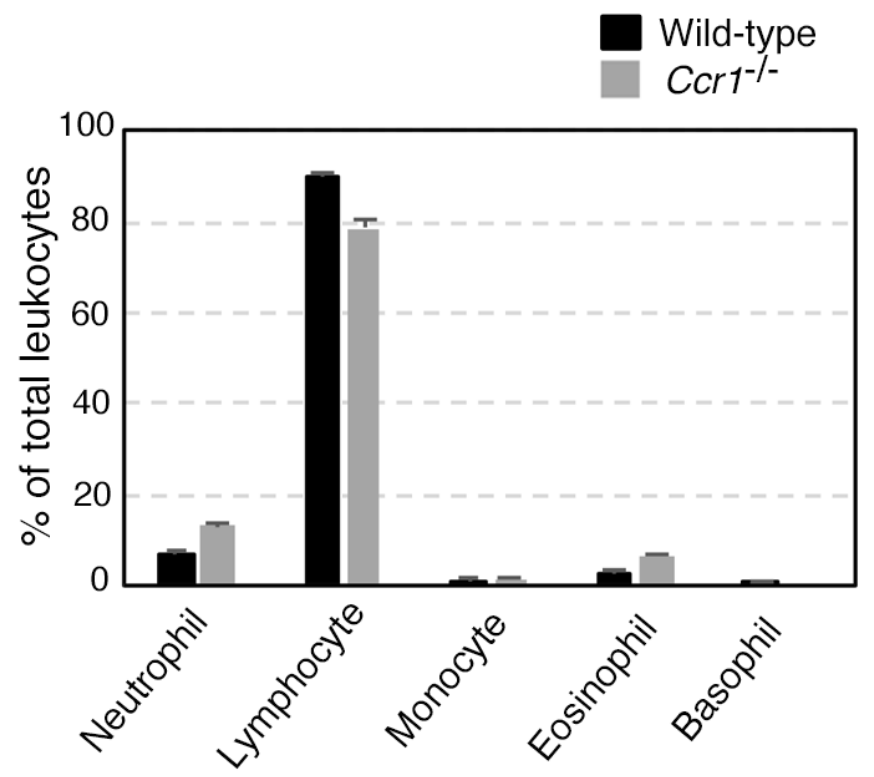

C

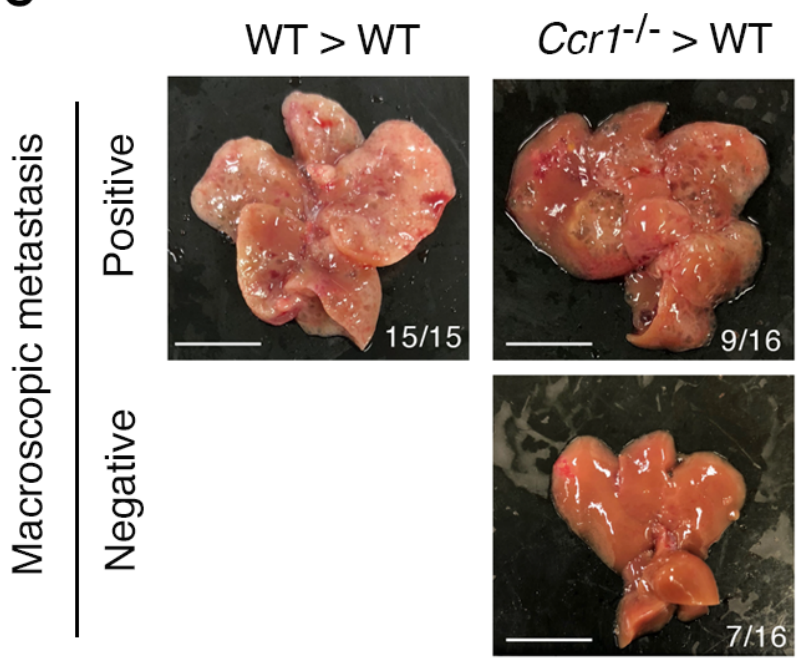

B

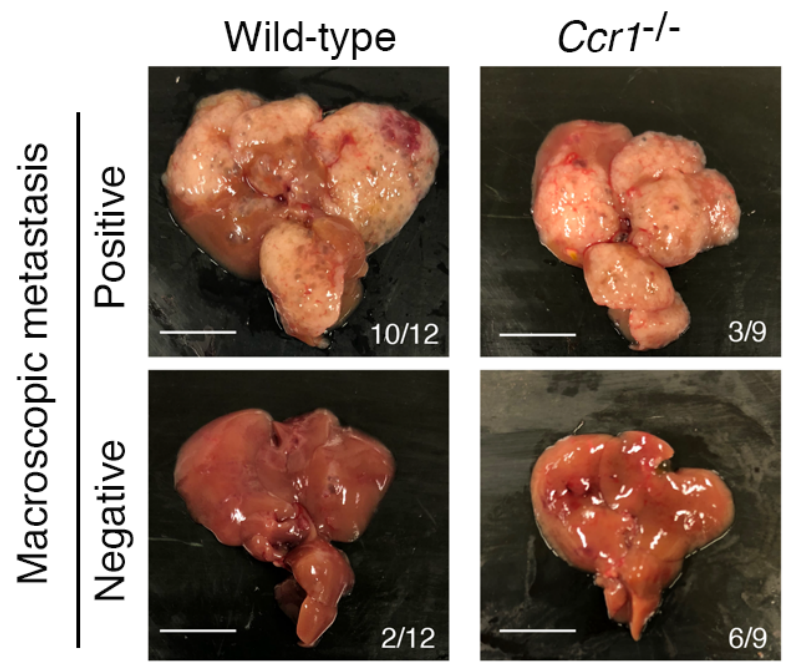




\section{Supplementary Fig.2}

A

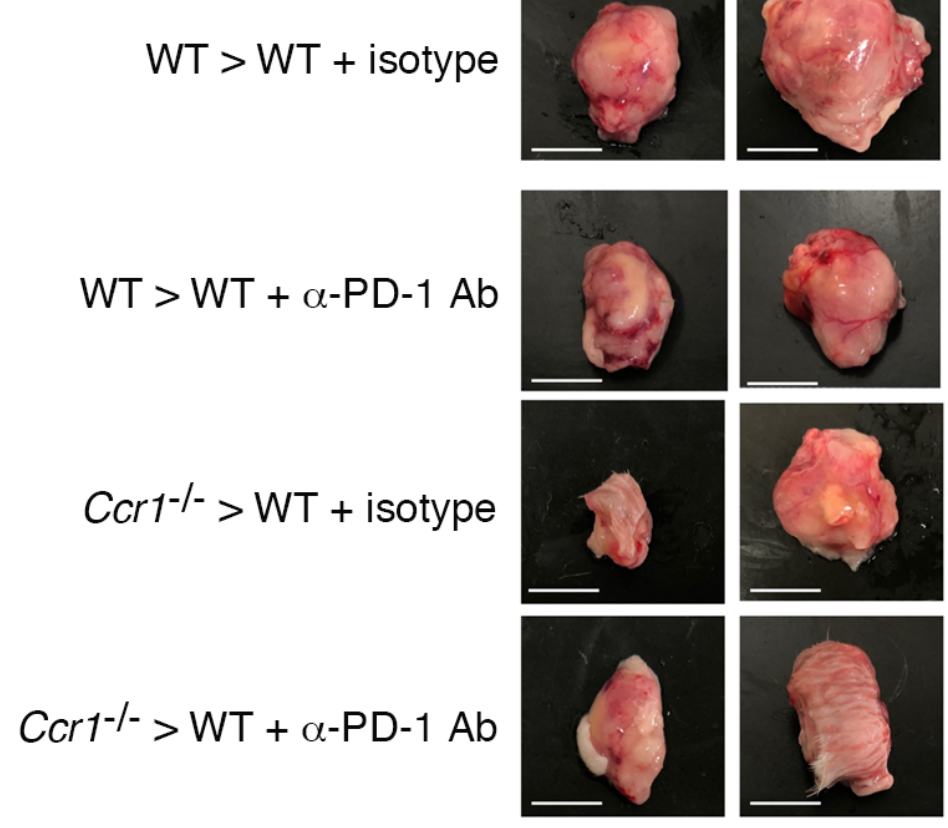

B

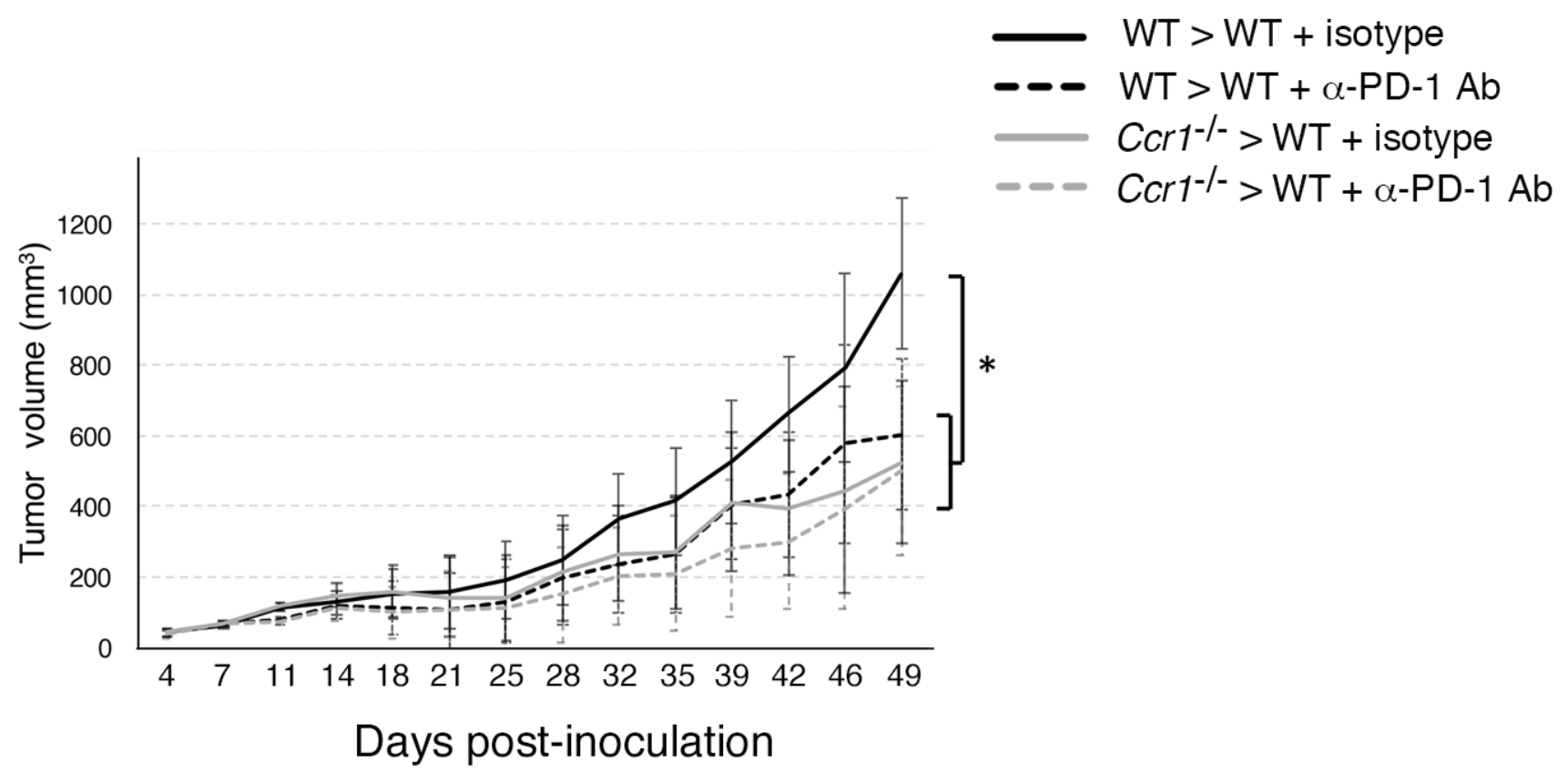




\section{Supplementary Fig.3}
